\title{
LARGE DOUBLY TRANSITIVE ORBITS ON A LINE
}

\author{
ALESSANDRO MONTINARO \\ (Received 4 November 2005; revised 1 June 2006)
}

Communicated by L. Batten

\begin{abstract}
Projective planes of order $n$ with a collineation group admitting a 2-transitive orbit on a line of length at least $n / 2$ are investigated and new examples are provided.

2000 Mathematics subject classification: primary 51E15; secondary $20 \mathrm{~B} 25$.
\end{abstract}

\section{Introduction}

A classical subject in finite geometries is the investigation of a finite projective plane $\Pi$ of order $n$ admitting a collineation group $G$ which acts 2-transitively on a pointsubset $\mathscr{O}$ of size $v$ of $\Pi$. It dates back to 1967 and it is due to Cofman [9]. It is easily seen that either

(i) the structure of a non trivial $2-(v, k, 1)$ design is induced on $\mathscr{O}$, or

(ii) $\mathscr{O}$ is an arc, or

(iii) $\mathscr{O}$ is a contained in a line.

This paper focus entirely on the case when $\mathscr{O}$ is a contained in a line. Starting from Cofman [10], several papers have been devoted to this case. In [10], Cofman proves that $\Pi$ is Desarguesian and $S L(2, n) \unlhd G$, under the assumptions that $v=n+1$ (that is, $\mathscr{O}$ is the entire line), $n \not \equiv 1 \bmod 8$ and $G$ contains involutory homologies. Some years later, Schulz [56] and Czerwinski [13] essentially proved that the unique translation planes with a collineation group acting 2-transitively on the line at infinity are either Desarguesian or Lüneburg planes. Actually, they proved this characterization under additional assumptions that ruled out the possibility for $G$ to contain Baer

(c) 2007 Australian Mathematical Society $1446-7887 / 07 \$ A 2.00+0.00$ 
collineations. Later, such additional assumptions were totally dropped with the use of the classification of finite 2 -transitive groups. In 1981, Korchmáros [44] investigated the general case $v=n+1$. when $n=2^{r}$. Apart from the Desarguesian case, the author proves that either $G \cong S z(n)$ or $G \cong P S U(3, n)$.

Also the case $v=n$ has been investigated extensively. In 1986, Ganley and Jha [19] proved that if $v=n$ and $\Pi$ is a translation plane and $l$ is the line at infinity, then $\Pi$ is actually a semifield plane. The case $v=n$ was investigated by Hiramine [29] in 1993 , without any assumption on the structure of $\Pi$. Apart from a few numerical values of $n$, Hiramine shows; that the socle of $\bar{G}$, where $\bar{G}$ denotes the group induced by $G$ on $\mathscr{O}$, is an elementary abelian $p$-group for some prime $p$, the plane $\Pi$ has order $n=p^{r}$ and either $\bar{G}_{O} \leq \Gamma L\left(1, p^{r}\right)$ or $S L\left(2, p^{r}\right) \leq \bar{G}_{O} \leq \Gamma L\left(2, p^{r}\right)$. In 1999, Biliotti, Jha and Johnson cla ssified the translation planes $\Pi$ for $v=n, n \neq 2^{6}$, when $l$ is the line at infinity and $\bar{G} \leq A \Gamma L\left(1, p^{r}\right)$. In 2000, Ganley, Jha and Johnson [20] classified the triple $\left(\Pi, \mathscr{O}, G_{\boldsymbol{\xi}}\right)$ for $v=n$, when $\Pi$ is a translation plane, $l$ is an affine line and $G$ is non solvable. Recently, Biliotti and Francot [4] investigated the general case $v \geq n$, determining all the possible collineation groups.

The problem of classifying the triple $(\Pi, \mathscr{O}, G)$ when $\mathscr{O} \subset l$ and the length $v$ of $\mathscr{O}$ is smaller than $n$, but close to $n$, is open. An initial result in this direction is the paper of Biliotti and Montinaro [8] devoted to the case $v=n-3$. In that paper no nontrivial cases arise.

The aim of this paper is to investigate the finite projective planes $\Pi$ of order $n$ admitting a collineation group $G$ which acts 2 -transitively on a subset $\mathscr{O}$ of a line $l$ of $\Pi$, under the assumption $v \geq n / 2$. In particular the following results are obtained.

THEOREM 1.1. Let $\Pi$ be a projective plane of order $n$ and let $\mathscr{O}$ be a 2-transitive $G$-orbit of length $v$ on a line. If $v \geq n / 2$ and $G$ is almost simple then one of the following occurs:

(1) $v=n+1$, and one of the following occurs:

(a) $n=q, \Pi \cong P G(2, q)$ and $S L(2, q) \unlhd G$;

(b) $n=q^{2}, q=2^{2 s+1}, s \geq 1$, and $S z(q) \unlhd G$;

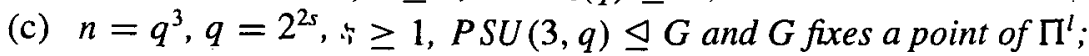

(2) $v=(n+1) / 2$, $n$ odd, and one of the following occurs:

(a) $\Pi$ is the Hall plane of order 9 or its dual, $|\mathscr{O}|=5$ and $S L(2,5) \unlhd G$;

(b) $n=2 q+1, q \equiv 3 \bmod 4, q \neq 7,|\mathscr{O}|=q+1$ and $S L(2, q) \unlhd G$;

(3) $v=n / 2, n$ even, and one of the following occurs:

(a) $\Pi$ is the Johnson-Walker translation plane of order 16 or its dual, and $P S L(2,7) \unlhd G$;

(b) $n=2(q+1), q \equiv 3 \bmod 4,|\mathscr{O}|=q+1$ and $S L(2, q) \unlhd G$. 
We remark that the result (1) is already known (see [4] and its references for related examples). So, our task is to prove the results (2) and (3). We also remark that there are no known examples for the cases ( $2 b)$ and $(3 b)$.

THEOREM 1.2. Let $\Pi$ be a projective plane of order $n$ and let $\mathscr{O}$ be a 2-transitive $G$-orbit of length $v$ on a line. If $v \geq n / 2$ and $G$ is of affine type then one of the following occurs:

(I) $v=n+1, n$ even, and either

(a) $\bar{G}_{O} \leq \Gamma L(1, v)$, or

(b) $v \in\left\{5^{2}, 7^{2}, 11^{2}, 19^{2}, 23^{2}, 29^{2}, 59^{2}\right\}$;

(II) $v=n$ and either

(a) $\bar{G}_{O} \leq \Gamma L(1, v)$, or

(b) $S L\left(2, p^{d / 2}\right) \unlhd \bar{G}_{o}, d$ even, or

(c) $v \in\left\{2^{4}, 3^{2}, 3^{4}, 3^{6}, 5^{2}, 7^{2}, 11^{2}, 19^{2}, 23^{2}, 29^{2}, 59^{2}\right\}$;

(III) $n / 2 \leq v<n$ and either

(a) $\bar{G} \leq A \Gamma L(1, v)$, or

(b) $v \in\left\{2^{4}, 2^{6}, 3^{2}, 3^{3}, 3^{4}, 3^{6}, 5^{2}, 7^{2}, 11^{2}, 19^{2}, 23^{2}, 29^{2}, 59^{2}\right\}$.

We remark that the results (I) and (II) are already known (see [4] and [29] for related examples). So we have to prove the result (III). We stress that, while there are no known examples for the case (IIIb), examples of type (IIIa) occur in the Desarguesian planes of order 8 and 9, in the Lorimer-Rahilly plane of order 16 and in the JohnsonWalker plane of order 16 and in their duals. A complete description of these examples is given in section 3 .

Clearly, Theorems 1.1 and 1.2 together cover all possibilities for a 2-transitive collineation group $G$.

The present paper is structured as follows. In section 2 we fix notation and the background of the problem and we recall some results which are useful for proving Theorems 1.1 and 1.2. In section 3, a complete description of the examples provided in the paper is given. In section 4 , we give some preliminary reductions for the structure of the 2-transitive collineation group $G$. Sections 5 and 6 are devoted to the proofs of Theorems 1.1 and 1.2, respectively. Finally, in section 7, our main problem is investigated under the additional assumption that $\Pi$ is the projective extension of a translation plane.

\section{Background}

The group-theoretical and geometrical notation used in this paper is standard. For the required background concerning finite groups, the reader is referred to $[1,22]$ 
and [34]. In particular, for the finite groups admitting a 2-transitive permutation representation we have the following classification.

THEOREM 2.1. Let $H$ be a finite group with a 2-transitive permutation representation of degree $v$ and let $S=\operatorname{soc}(H)$ be the socle of $H$. Then one of the following occurs:

(1) $S$ is non abelian simple, and $S \leq H \leq$ Aut $S$ where $S$ and $v$ are as follows:

(a) $A_{v}$ with $v \geq 5$;

(b) $\operatorname{PSL}(d, q), d \geq 2, v=\left(q^{d}-1\right) /(q-1)$ and $(d, q) \neq(2,2),(2,3)$;

(c) $P S U(3, q), v=q^{3}+1, q>2$;

(d) $S z(q), v=q^{2}+1, q=2^{2 e+1}>2$;

(e) ${ }^{2} G_{2}(q)^{\prime}, v=q^{3}+1, q=3^{2 e+1}$;

(f) $\operatorname{Sp}(2 n, 2), n \geq 3, v=2^{2 n-1} \pm 2^{n-1}$;

(g) $\operatorname{PSL}(2,11), v=11$;

(h) Mathieu groups $M_{v}, v=11,12,22,23,24$;

(i) $M_{11}, v=12$;

(j) $A_{7}, v=15$;

(k) $H S$ (Higman-Sims group), $v=176$;

(l) $\mathrm{Co}_{3}$ (Conway's smallest group), $v=276$.

(2) $S$ is an elementary abelian group of order $v=p^{d}$, where $p$ is a prime. Identify $G$ with a group of affine transformations $x \longmapsto x^{g}+c$ of $G F(p)^{d}$, where $g \in G_{0}$. Then one of the following occurs:

(a) $G_{0} \leq \Gamma L\left(1, p^{d}\right)$;

(b) $S L(a, q) \unlhd G_{0}$, where $a \geq 2$ and $q^{a}=p^{d}$;

(c) $S p(a, q) \unlhd G_{0}$, where $a \geq 4$, a even, and $q^{a}=p^{d}$;

(d) $G_{2}(q)^{\prime} \unlhd G_{0}$ where $q^{6}=p^{d}$ and $q$ is even;

(e) $G_{0} \cong A_{6}$ or $A_{7}, p^{d}=2^{4}$;

(f) $S L(2,3) \unlhd G_{0}$ or $S L(2,5) \unlhd G_{0}, v=p^{2}$ and $p=5,7,11,19,23,29$, or 59 , or $v=3^{4}$;

(g) $G_{0}$ has a normal extraspecial subgroup $R$ of order $2^{5}$ and $G_{0} / R \leq S_{5}$;

(h) $G_{0} \cong S L(2,13), p^{d}=3^{6}$.

See for example [40].

A finite 2-transitive group is said either almost simple or of affine type according to whether its socle is a nonabelian simple or an elementary abelian $p$-group for some prime $p$, respectively.

The background concerning finite projective planes may be found in [33]. Let $\Pi=(\mathscr{P}, \mathscr{L})$ be a finite projective plane of order $n$. If $G$ is a collineation group and $P \in \mathscr{P}(l \in \mathscr{L})$, we denote by $G(P)$ (by $G(l))$ the subgroup of $G$ consisting of perspectivities with the centre $P$ (the axis $l$ ). Also, $(P, l)=G(P) \cap L(l)$. 
Furthermore, we denote by $G(P, P)$ (by $G(l, l)$ ) the subgroup of $G$ consisting of elations with the centre $P$ (the axis $l$ ).

The following theorems deal with projective planes $\Pi$ of order $n$ with a collineation group $G$ acting 2-transitively either on the points of a line, or on the points of a line minus one.

THEOREM 2.2. Let $\Pi$ be a projective plane of order $n$ with a collineation group $G$ acting 2-transitively on the points of a line. Then one of the following occurs:

(1) $\Pi \cong P G(2, n)$ and $S L(2, n) \unlhd G$;

(2) $n=q^{2}, q=2^{2 s+1}, s \geq 1$, and $S z(q) \unlhd G$;

(3) $n=q^{3}, q=2^{2 s}, s \geq 1, P S U(3, q) \unlhd G$ and $G$ fixes a point of $\Pi^{l}$;

(4) $n=p^{h}-1, p$ an odd prime, and $G \leq A \Gamma L(1, v)$;

(5) $n=p^{h}-1, p^{h} \in\left\{5^{2}, 7^{2}, 11^{2}, 19^{2}, 23^{2}, 29^{2}, 59^{2}\right\}$, and $G^{l}$ is sharply transitive on l except possibly for $p^{h}=5^{2}$ or $29^{2}$.

For a proof see [4, Theorems 5.2 and 5.5].

Note that, while the there no known examples corresponding to the cases (3)-(5), the case (2) really occurs in the projective extensions of the Lüneburg planes.

We now consider the case where $G$ fixes an incident point-line pair $(L, l)$ of $\Pi$ and acts 2-transitively on $l-\{L\}$.

THEOREM 2.3 (Hiramine). Let $\Pi$ be a projective plane of order $n$ with a collineation group $G$ that fixes an incident point-line pair $(L, l)$ of $\Pi$ and acts 2-transitively on $l-\{L\}$. Then $n=p^{d}, p$ prime, and $\bar{G}$ contains a normal elementary abelian $p$-group acting regularly on $l-\{L\}$. In particular, one of the following occurs:

(1) $\bar{G}_{o} \leq \Gamma L\left(1, p^{d}\right)$;

(2) $S L\left(2, p^{d / 2}\right) \unlhd \bar{G}_{o}, d$ even;

(3) $p^{d} \in\left\{2^{4}, 3^{2}, 3^{4}, 3^{6}, 5^{2}, 7^{2}, 11^{2}, 19^{2}, 23^{2}, 29^{2}, 59^{2}\right\}$.

In the following result, the two previous situations are analyzed under the further assumption that $\Pi$ is the projective extension of a translation plane.

THEOREM 2.4. Let $\Pi$ be the projective extension of a translation plane of order $n$ and let $(L, l)$ be an incident point-line pair of $\Pi$, and let $G$ be a collineation group of $\Pi$ fxing the line $l$. Then one of the following occurs:

(1) If $G$ acts 2-transitively on $l$ then $l$ is the line at infinity and either

(a) $\Pi$ is Desarguesian, or

(b) $\Pi$ is a Lüneburg plane.

(2) If $n \neq 2^{6}, G$ fixes the point $L$ and acts 2-transitively on $l-\{L\}$, where $l$ is the line at infinity, then either 
(a) $\Pi$ is a Desarguesian, or

(b) $\Pi$ is a Generalized Twisted Field plane.

(3) If $n \notin\left\{3^{4}, 3^{6}, 11^{2}, 19^{2}, 29^{2}, 59^{2}\right\}, G$ is non solvable, $G$ fixes the point $L$ and acts 2-transitively on $l-\{L\}$, wherel is an affine line, then either

(a) $\Pi$ is Desarguesian, or

(b) $\Pi$ is one of the three Walker planes of order 25 , or

(c) $\Pi$ is the Dempwolff plane of order 16.

See for example [38, Theorem 4.3.16] for a proof of the case (1), see [5, Theorem 8.1] for a proof of the case (2) and see [20, main theorem] for a proof of the case (3). Clearly all these cases really occur. Note that a classification of the projective extensions of translation planes, when $l$ is an affine line and one of the situations (1) or (3) of Hiramine's theorem occurs, is not available. Nevertheless, there are several examples corresponding to each of these situations (see [6]). In particular, in the examples referring to the situation (3), $A_{5}$ is involved in $\bar{G}_{O}$ in many cases.

\section{Examples}

In this section we provide some examples. It is worth noting that, while Examples 1 and 2 are already known, Example 3 is new.

EXAMPLE 1. Let $\Pi$ be a projective plane of order $n$, with $n \leq 9$, and let $G$ be a collineation group of $\Pi$. Suppose that $G$ induces a group $\bar{G}$ which has a 2 -transitive point-orbit of length $v$ on a line $l$. If $n>v \geq n / 2$, then one of the following occurs:

(1) $\Pi \cong P G(2,4), \bar{G} \cong A G L(1,3)$ and there is exactly one 2 -transitive $\bar{G}$-orbit of length 3 on $l$;

(2) $\Pi \cong P G(2,5), \bar{G} \cong A G L(1,3)$ and there are exactly two 2 -transitive $\bar{G}$-orbits of length 3 on $l$;

(3) $\Pi \cong P G(2,7), G \cong S L(2,3)$, in particular $\bar{G} \cong A G L(1,4)$ and there are exactly two 2 -transitive $\bar{G}$-orbits of length 4 on $l$;

(4) $\Pi \cong P G(2,8), \bar{G} \cong A G L(1,4)$ and there are exactly two 2 -transitive $\bar{G}$-orbits of length 4 on $l$;

(5) $\Pi \cong P G(2,8), \bar{G} \cong A G L(1,7)$ and there is exactly one 2 -transitive $\bar{G}$-orbit of length 7 on $l$;

(6) $\Pi \cong P G(2,9), \bar{G} \cong A G L(1,5)$ and there are exactly two 2-transitive $\bar{G}$-orbits of length 5 on $l$;

(7) $\Pi \cong P G(2,9), G \cong S L(2,5)$, in particular $\bar{G} \cong A_{5}$ there are exactly two 2-transitive $\bar{G}$-orbits of length 5 on $l$. 
In particular, each of these cases really occurs.

Let $\Pi$ be a projective plane of order $n$, with $n \leq 9$, and let $G$ be a collineation group of $\Pi$ inducing a group $\bar{G}$ which has a 2-transitive point-orbit of length $v$ on a line $l$. Assume that $n>v \geq n / 2$. Clearly $v \geq 3$.

If $v=3$ then $3<n \leq 6$. Actually, $n<6$ by [33, Theorem 3.6]. Hence either $n=4$ or $n=5$. If $\Pi \cong P G(2,4)$ then any subgroup $\bar{G}$ of $P \Gamma L(2,4)$ isomorphic to $D_{6} \cong A G L(1,3)$ and containing the involution induced by the Frobenius automorphism of $G F(4)$ fixes two points on $l$ and acts 2-transitively on the remaining ones. Thus (1). If $\Pi \cong P G(2,5)$ the group $S L(2,5)$ induces $A_{5}$ on $l$ and any $\bar{G} \cong D_{6}$ inside $A_{5}$ has two 2-transitive point-orbits on $l$ both of length 3 , and hence (2).

If $v=4$ then $4<n \leq 8$. That is $n=5,7$ or 8 . The are no examples in $P G(2,5)$, since the stabilizer in $P G L(2,5)$ of three distinct points on a line is trivial. In $P G(2,7)$ there is exactly one example: the group $G \cong S L(2,3)$ has two 2-transitive point-orbits on $l$, both of length 4 . Therefore (3). In $P G(2,8)$ there is exactly one example: a subgroup $\bar{G}$ of $P \Gamma L(2,8)$ isomorphic to $A G L(1,4)$ has two 2-transitive point-orbits on $l$, both of length 4 . Hence (4).

If $v=5$ then $5<n \leq 9$. If $n=7$ or 8 then $\Pi \cong P G(2, n)$. Nevertheless these cases cannot occur, since $5 \nmid|P \Gamma L(2, n)|$. Therefore $n=9$. Then either $\Pi$ is Desarguesian or $\Pi$ is one of the Hall planes by [58]. It is easily seen that there exists a subgroup $\bar{G} \cong A G L(1,5)$ of $P \Gamma L(2,9)$ splitting $l$ in two 2 -transitive orbits, both of length 5 when $\Pi$ is Desarguesian, and hence (6). If $\Pi$ is the Hall plane of order 9 then the group induced on the line at infinity by the full translation complement of $\Pi$ is described in the proof of Lemma 5.2 of [18]. It is easy to check with [24], by using such a description, that the group induced on the line at infinity does not contain solvable subgroups with a 2-transitive permutation representation of degree 5 . Nevertheless, the group $G \cong S L(2,5)$ induces $A_{5}$ on the line at infinity and this one is split in two 2-transitive $A_{5}$-orbits, both of length 5 (see [6]). Thus (7).

If $v=6$ then $6<n \leq 9$. If $n=7$ or 8 then $\Pi \cong P G(2, n)$. Thus $\bar{G} \leq P \Gamma L(2, n)$ such that $\bar{G}$ is 2 -transitive orbit on $l$ of length 6 . Clearly 5|| $\bar{G} \mid$. This gives a contradiction, since $5 \nmid|P \Gamma L(2, n)|$ for $n=7$ or 8 . So $n=9$. Nevertheless, this case cannot occur by [8, Theorems 25 and 35], since $n-v=3$.

If $v=7$ then $7<n \leq 9$. That is $n=8$ or $n=9$. If $n=9$ then $\Pi \cong P G(2,9)$ by [57, Lemma 8.2$]$. So $\bar{G} \leq P \Gamma L(2,9)$. This gives a contradiction since 7|| $\bar{G} \mid$ while $7 \nmid|P \Gamma L(2,9)|$. Thus $n=8$ and hence $\Pi \cong P G(2,8)$. Let $l$ be a line of $\Pi \cong P G(2,8)$. Clearly $P \Gamma L(2,8)$ acts on $l$. Pick any $Z_{7}$ in $P \Gamma L(2,8)$. Then $Z_{7}$ fixes two points $P_{1}$ and $P_{2}$ on $l$ and $Z_{7}$ acts regularly on $l-\left\{P_{1}, P_{2}\right\}$. Furthermore, $N_{P \Gamma L(2,8)}\left(Z_{7}\right) \cong D_{14} \cdot\langle\sigma\rangle$, where $\sigma$ is the collineation of $\Pi$ "induced" by a Frobenius automorphism of $G F(8)$. Set $\mathscr{O}=l-\left\{P_{1}, P_{2}\right\}$ and $\bar{G} \cong N_{P \Gamma L(2,8)}\left(Z_{7}\right)$. Then $\bar{G} \cong A G L(1,7)$ acts 2 -transitively on $\mathscr{O}$ and $v=7$. Thus (6). 
If $v=8$ then $n=9$. Then $\Pi \cong P G(2,9)$ by $[57$, Lemma 8.2], since 7|| $\bar{G} \mid$ as $\bar{G}$ acts 2-transitively on $\mathscr{O}$ and $v=8$. This gives a contradiction, as above.

EXAMPLE 2. In the Johnson-Walker translation plane $\Pi$ of order 16 or its dual, there exists an affine line $l$ on which a group $G$ isomorphic to $\operatorname{PSL}(2,7)$ has two 2-transitive orbits of length 7 and 8.

This example follows by [15, Theorems $4.8-4.10$ and Section 5].

EXAMPLE 3. In the Lorimer-Rahilly translation plane of order 16 , in the JohnsonWalker translation plane of order 16 and in their duals, the group $G \cong A G L(1,8)$ admits a 2 -transitive orbit $\mathscr{O}$ of length 8 on a line.

Let $\Pi$ be the Lorimer-Rahilly translation plane of order 16 or the Johnson-Walker translation plane of order 16 or one of their duals. Denote by $l_{\infty}$ the line at infinity of $\Pi$. Let $T$ be the full translation group of $\Pi$ and let $H \cong Z_{7}$ be a subgroup of the translation complement fixing the point $O$ of $\Pi$. Clearly $H$ fixes a point $P$ on $l_{\infty}$. Let $T_{1}$ be the subgroup of translations of $\Pi$ of direction $P$. Then $T_{1}$ fixes the line $P O$ and it acts regularly on $P O-\{P\}$. Furthermore, $H$ acts on $T_{1}-\{1\}$ and on $P O-\{P, O\}$ in the same way, by [51, Proposition 4.2]. In particular, $H$ leaves a subgroup $T_{2}$ of $T_{1}$ of order 8 invariant, since $T_{0}-\{1\} \cong P G(3,2)$ and $Z_{7}$ fixes exactly a non incident point-plane pair in $P G(3,2)$ by [50, Table I]. Clearly $Z_{7}$ is transitive on $T_{1}-\{1\}$. Set $\mathscr{O}=O^{T_{1}}$ and $G \cong T_{2}$.H. Then $G \cong A G L(1,8)$ acts 2-transitively on $\mathscr{O}$ and $|\mathscr{O}|=8$.

We remark that in the other known projective planes of order 16 there are no examples of 2-transitive orbits of length 8 on a line (see [52, Table 1]).

\section{Preliminaries}

Let $G$ be a collineation group having an orbit $\mathscr{O}$ of points of $\Pi$ on which $G$ acts 2transitively. We call $\mathscr{O}$ a 2 -transitive $G$-orbit, or just a 2-transitive orbit. Furthermore, we say that $\mathscr{O}$ is non trivial if $|\mathscr{O}|>1$. Note that $v \geq 3$ since $G$ is 2 -transitive. In what follows, we assume that $v \geq 5$. It is a plain that $n \geq 8$.

The following numerical and group-theoretical lemmas will be useful hereafter.

LEMMA 4.1. Let $t^{j}, j \geq 0$, and $p^{r}, r \geq 0$, be two powers of primes such that $p^{r} \equiv 3 \bmod 4$. Then the following holds:

(1) If $t^{j}=2 p^{r}+1$ then $j=1$.

(2) If $t^{j}=2\left(p^{r}+1\right)$ then $t=2, r=1$ and $p$ is a Mersenne prime. 
PROOF. The assertion (2) follows by [54, Result (B1.1)], since $t=2$. Hence, assume that $t^{j}=2 p^{r}+1$. Then $t^{j} \equiv 3 \bmod 4$, since $p^{r} \equiv 3 \bmod 4$. Thus $t \equiv 3 \bmod 4$ and $j$ is odd. In particular $2 p^{r}=(t-1)\left[\left(t^{j}-1\right) /(t-1)\right]$. Hence $2 p^{r-h}=t-1$ and $p^{h}=\left(t^{j}-1\right) /(t-1), 0 \leq h \leq r$, since $t$ and $j$ are odd. Assume that $0<h<r$. Then $p \mid \operatorname{gcd}\left(t-1,\left(t^{j}-1\right) /(t-1)\right)$. Hence $p \mid j$ by [54, Result P1.2(ii)]. But this contradicts [54, Result A8.5(1)]. Assume that $h=r$. Then $t=3$ and $\left(3^{j}-1\right) /(3-1)=p^{r}$. Then $(p, r, j)=(11,2,3)$ by [60, Theorem 3]. This gives a contradiction, since $p^{r}=11^{2}$ and $11^{2} \not \equiv 3 \bmod 4$. Hence $h=0$ and $j=1$. This proves the assertion (1).

A class of solutions to the first Diophantine equation is furnished by the SophieGermain primes $(r=1)$ (see [55]).

Denote by $d_{j}(H), j \geq 0$, the primitive permutation representation degrees of a group $H$ in increasing order, so $d_{0}(H)$ denotes the minimal one. If $v$ is a 2-transitive permutation representation degree of $H$ then $d_{0}(H) \leq v$.

LEMMA 4.2. Let $H$ be a 2-transitive non-abelian simple group such that $d_{0}(H)=v$. Then either $d_{j}(L)>d_{0}(L)+\sqrt{2 d_{0}(L)}$ for $j>0$, or $d_{1}(L)=d_{0}(L)+1$ and one of the following occurs:

(1) $H \cong A_{5}$ and $v=5$;

(2) $H \cong P S L(2,7)$ and $v=7$;

(3) $H \cong P S L(2,11)$ and $v=11$;

(4) $H \cong M_{11}$ and $v=11$.

PROOF. The assertion is true when $H$ is sporadic by a direct inspection of [11]. Elementary calculations with [59] and with [42] show that the assertion is also true when $H$ is exceptional of Lie type. When $H$ is alternating, the assertion follows by a straightforward calculation by [34, Satz IV.4.6] for $v \geq 9$ and by [11] for $5 \leq v<9$. Assume that $H$ is simple classical group. Then the assertion follows by [34, Haupsatz II.8.27] when $H \cong P S L(2, q)$. Furthermore, the assertion follows by [25] and [49] when $H \cong P S L(3, q)$. It remains to resolve the cases $H \cong P S L(d, q)$, $d \geq 4$, and $H \cong S p(d, 2), d=2 h$ and $h \geq 3$, by the list given in Theorem 2.1. As a consequence of the structure theorems given in Kleidman and Liebeck [43], every maximal subgroup of $H$ lies in the classes $\cup_{i=1}^{8} \mathscr{C}_{i}$ or in the class $\mathscr{S}$, where the structure of every member of $\mathscr{C}_{i}$ is shown in [43, Tables 3.5.A-C]. Let $M$ be a maximal subgroup of $H$ such that $[H: M]>d_{0}(H)$. If $M \in \mathscr{S}$, then $|M| \leq q^{3 d}$ by [46]. Then $[H: M] \geq 2 v+1$ by an easy calculation. If $M \in \cup_{i=1}^{8} \mathscr{C}_{i}$ then a straightforward calculation of $[H: M]$ with [43, Tables 3.5.A-C], with the structure proposition members of $\mathscr{C}_{i}$ given in [43, Chapter 4], in conjunction with [12] and with Lemma 4 and Table II of [45], shows that the assertion is true also in this case. 
LEMMA 4.3. Let $H$ be a 2-transitive non-abelian simple group such that $d_{0}(H)<v$. Then the following holds:

(1) $d_{j}(H)>v+\sqrt{2 v}$ for $j>1$;

(2) $2 d_{0}(H)>v+1$, except

(a) $H \cong A_{7}$, where $2 d_{0}(H)=v-1$ and $v=15$;

(b) $H \cong A_{8}$, where $2 d_{0}(H)=v+1$ and $v=15$;

(c) $H \cong P S U(3,5)$, where $2 d_{0}(H)=v-26$ and $v=126$.

Proof. In Table 1 the non abelian simple groups such that $d_{0}(H)<v$ are listed (see Theorem 2.1):

TABLE 1 .

\begin{tabular}{|l|l|l|}
\hline$P S L(2,5)$ & $v=6$ & $d_{0}(H)=5$ \\
\hline$P S L(2,7)$ & $v=8$ & $d_{0}(H)=7$ \\
\hline$P S L(2,9)$ & $v=10$ & $d_{0}(H)=6$ \\
\hline$P S L(2,11)$ & $v=12$ & $d_{0}(H)=11$ \\
\hline$P S U(3,5)$ & $v=126$ & $d_{0}(H)=50$ \\
\hline$A_{7}$ & $v=15$ & $d_{0}(H)=7$ \\
\hline$A_{8}$ & $v=15$ & $d_{0}(H)=8$ \\
\hline$M_{11}$ & $v=12$ & $d_{0}(H)=11$ \\
\hline$H S$ & $v=176$ & $d_{0}(H)=100$ \\
\hline$S p(2 h, 2), h \geq 3$ & $v=2^{h-1}\left(2^{h}+1\right)$ & $d_{0}(H)=2^{h-1}\left(2^{h}-1\right)$ \\
\hline
\end{tabular}

The assertion (1) easily follows by a direct inspection in [11] of the primitive permutation representations of the groups $H$ listed in Table 1 and not isomorphic to $S p(2 h, 2), h \geq 3$. When $H \cong S p(2 h, 2), h \geq 3$, a similar argument to that in Lemma 4.2, $H$ being classical, also proves the assertion in this case. Now the assertion (2) can be easily read off from Table 1

Let $N$ be the kernel of the action of $G$ on $\mathscr{O}$ and set $\bar{G}=G / N$. We may also assume that $G$ is the minimal preimage of $\bar{G}$. We now present some preliminary reductions for the structure of $N$.

LEMMA 4.4. $N=\Phi(G)$, where $\Phi(G)$ is the Frattini subgroup of $G$.

Proof. Let $S$ be any Sylow $t$-subgroup of $N$. Then $G=N_{G}(S) N$ by Frattini's argument. Thus $S \triangleleft G$ by the minimality of $G$. Therefore $N$ is nilpotent. Suppose that $N \leftleftarrows \Phi(G)$. Then there exists a maximal subgroup $M$ of $G$ such that $G=N M$ by [34, Satz 3.2(b)]. Clearly $M<G$ and $M /(M \cap N) \cong \bar{G}$. This contradicts the minimality of $G$. Hence we may assume that $N \leq \Phi(G)$. Note that $G_{P}$ is 
maximal in $G$ for each point $P \in \mathscr{O}$, since $N \triangleleft G_{P}$ and $\bar{G}$ is primitive on $\mathscr{O}$. Hence $\Phi(G) \triangleleft G_{P}$ for each point $P \in \mathscr{O}$. Therefore $N=\Phi(G)$.

LEMMA 4.5. If $N \neq\langle 1\rangle$, then one of the following occurs:

(1) $G$ fixes a unique point $Q$ on $\Pi-l, N$ is semiregular on $\Pi-(l \cup\{Q\})$ and $|N| \mid n-1$

(2) $N$ is semiregular on $\Pi-l$ and $|N| \mid n^{2}$. In particular one of the following occurs:

(a) $|N| \mid n$ and $N$ is semiregular on $[Y]-\{l\}$ for any point $Y \in \mathscr{O}$;

(b) $n|| N \mid, n=u^{j}, j \geq 1, N$ is a u-group and $\left[N: N_{a}\right]=n$ for any line a of $\Pi$ intersecting $l$ in $\mathscr{O}$;

(c) $n|| N \mid, n=2^{j+1}, j>1, N$ is a 2-group and $\left[N: N_{a}\right]=n / 2$ for any line $a$ of $\Pi$ intersecting $l$ in $\mathscr{O}$;

(d) $|N|>n, n=3|S| / 2, S$ is a Sylow 2-subgroup of $N$ and $S \leq N \leq S \times A$, where $A$ is a group of order a divisor of 9 .

PROOF. Suppose there exists $\alpha \in N, \alpha \neq 1$, such that $\alpha$ is planar on $\Pi$. Then $o(\operatorname{Fix}(\alpha)) \geq n / 2-1$, since $\alpha$ fixes $\mathscr{O}$ pointwise and $v \geq n / 2$. So $(n / 2-1)^{2} \leq n$ by [33, Theorem 3.7]. This gives a contradiction, since $n \geq 8$. Thus $N$ does not contain any non trivial planar collineation of $\Pi$.

Assume that there exists an element $\sigma \in N, \sigma \neq 1$, such that $\sigma$ fixes a point $P$ of $\Pi-l$. Actually, $P$ is the unique point on $\Pi-l$ fixed by $\sigma$ since $N$ does not contain any non trivial planar collineation of $\Pi$. Furthermore, $P$ is the unique point on $\Pi-l$ fixed by $Z(N)$. Thus $G$ fixes $P$, since $Z(N) \triangleleft G$ and $Z(N) \neq\langle 1\rangle$ being $N$ nilpotent by Lemma 4.4. This proves the assertion (1).

Assume that $N$ is semiregular on $\Pi-l$. Hence $|N| \mid n^{2}$. If $N$ is semiregular on $\left[Y_{0}\right]-\{l\}$, for some point $Y_{0} \in \mathscr{O}$, then $|N| \mid n$ and $N$ is semiregular on $[Y]-\{l\}$ for any point $Y \in \mathscr{O}$, since $G$ is transitive on $\mathscr{O}$. This proves the result (2a).

Assume that there exists $b_{X} \in[X]-\{l\}$ such that $N_{b_{X}} \neq\langle 1\rangle$ for each $X \in \mathscr{O}$. If $N_{e} \cap N_{c} \neq\langle 1\rangle$ for some couple of lines $e$ and $c$ intersecting $l$ in distinct points of $\mathscr{O}$, then there exists $\gamma \in N, \gamma \neq 1$, fixing the point $e \cap c$ which lies in $\Pi-l$. This gives a contradiction, since $N$ is semiregular on $\Pi-l$. Therefore we may assume that $N_{h} \cap N_{z}=\langle 1\rangle$, with $N_{h}, N_{z} \neq\langle 1\rangle$, for any couple of lines $h$ and $z$ intersecting $\mathscr{O}$ in distinct points. In particular, $N_{t}<N$ for each line $t$ of $\Pi$ intersecting $\mathscr{O}$. Thus $[P]-\{l\}$ consists of nontrivial $N$-orbits for any point $P$ of $\mathscr{O}$. Let $a \in[O]-\{l\}$, $O \in \mathscr{O}$, be such that $N_{a} \neq\langle 1\rangle$. Let $S_{a}$ be the Sylow $u$-subgroup of $N_{a}$, where $u$ is a prime dividing $\left|N_{a}\right|$, and let $S$ be the Sylow $u$-subgroup of $N$. Assume that $S=S_{a}$. Then $S_{a} \triangleleft G$ as $N$ is nilpotent. Let $g \in G$ be such that $O g \neq O$. Since $S_{a} \triangleleft G, S_{a}$ fixes the line $a g$ and hence the point $a \cap a g$ which lies in $\Pi-l$. This gives a contradiction, since $N$ is semiregular on $\Pi-l$. Therefore $S_{a}<S$. 
Furthermore, $|S|-1 \geq v\left(\left|S_{a}\right|-1\right)$ since $S_{a} \neq\langle 1\rangle, S \triangleleft G$ and $G$ is transitive on $\mathscr{O}$. Then $\left[S: S_{a}\right] \geq\left[v\left(\left|S_{a}\right|-1\right)+1\right] /\left|S_{a}\right|$. Hence either $\left|S_{a}\right| \geq 3$ and $\left[S: S_{a}\right]>2 v / 3$, or $\left|S_{a}\right|=2$ and $\left[S: S_{a}\right] \geq(v+1) / 2$. Denote by $k$ the number of $S$-orbits on $[O]-\{l\}$, where $O \in \mathscr{O}$. Arguing as above with $S$ in the role of $N$, as $S \triangleleft G$, we see that $[O]-\{l\}$ consists of nontrivial $S$-orbits. Let $x \in[O]-\{l\}$ be such that $\left|x^{S}\right| \leq\left|y^{S}\right|$ for any $y \in[O]-\{l\}$. Then $k\left|x^{S}\right| \leq n$.

Assume that $\left|S_{x}\right| \geq 3$. Then $k \leq 2$ as $\left|x^{s}\right|>2 v / 3$ and $n \leq 2 v$. If $k=1$ then $\left|x^{s}\right|=n$. Hence $n=u^{j}, j \geq 1$, as $\left|x^{s}\right|=u^{j}$. Thus $N=S$ as $|N| \mid n^{2}$, and we have the assertion (2b). Assume that $k=2$. Then $[O]-\{l\}=x^{S} \cup b^{S}$ for some line $b$ of $[O]-\left(\{l\} \cup x^{s}\right)$. Hence $n=\left|x^{s}\right|+\left|b^{s}\right|$. Assume that $\left|x^{s}\right|<\left|b^{s}\right|$. Then $\left|x^{S}\right| u \leq\left|b^{S}\right|$, since $S$ is a $u$-group. Hence $\left|b^{S}\right|>2 v u / 3$ as $\left|x^{S}\right|>2 v / 3$. Then $2 v u / 3+2 v / 3<n$, as $n=\left|x^{s}\right|+\left|b^{S}\right|$. This gives a contradiction, since $u \geq 2$ and $n \leq 2 v$. As a consequence $\left|x^{s}\right|=\left|b^{s}\right|$. Hence $n=2 u^{j}$ since $\left|x^{s}\right|=u^{j}$. Then $u=2$ and $n=2^{j+1}$ by [33, Theorem 13.18]. Then $N=S$ since $|N| \mid n^{2}$. In particular, $\left|x^{N}\right|=\left|b^{N}\right|=n / 2$. This proves the assertion (2c).

Assume that $\left|S_{x}\right|=2$. Then $k \leq 3$ as $\left|x^{s}\right| \geq(v+1) / 2$ and $n \leq 2 v$. Note that $S_{x}=S(x \cap l, l)$, since $\left|S_{x}\right|=2, S_{x}<N$ and $N$ cannot contain non trivial planar elements. Thus $\left|S_{y}\right| \geq 2$ and $\left|y^{S}\right| \leq\left|x^{S}\right|$ for any $y \in[O]-\{l\}$. Hence $\left|y^{S}\right|=\left|x^{S}\right|$, since $\left|x^{S}\right| \leq\left|y^{S}\right|$ for any $y \in[O]-\{l\}$. Then $n=k|S| / 2, k \leq 3$. If $k \leq 2$, then $N=S$ as $|N| \mid n^{2}$, and we again have the assertions (2b) and (2c). If $k=3$ then $n=3|S| / 2$. Hence $S \leq N \leq S \times A$, where $A$ is a group of order a divisor of 9 , since $|N| \mid n^{2}$. This proves the assertion (2d).

Recall that $\operatorname{soc}(\bar{G})$ denotes the socle of $\bar{G}$. Also, recall that either $\bar{G}$ is almost simple or of affine type, since $\bar{G}$ is 2 -transitive on $\mathscr{O}$. We treat these two cases separately.

\section{5. $\bar{G}$ is almost simple.}

Assume that $\bar{G}$ is almost simple. We treat the cases $N \neq\langle 1\rangle$ and $N=\langle 1\rangle$ separately.

5.1. The unfaithful case Assume that $N \neq\langle 1\rangle$. We continue investigating the structure of $N$.

LEMMA 5.1. If $\bar{G}$ is non abelian and simple, then one of the following holds:

(1) $G$ is a covering group for $\bar{G}$;

(2) There exists a Sylow $t$-subgroup $S$ of $N$ such that $\bar{G} \leq S L(V)$, where $V=$ $S / \Phi(S)$. In particular, $|S| \geq 1+d_{0}(\bar{G})$.

Proof. Assume that $N \leq Z(G)$. Then $G^{\prime}$ is a covering group for $\bar{G}$ by [1, 
Theorem 11.33.3], since $\bar{G}$ is a non abelian simple group. Furthermore, $G^{\prime}=G$ by the minimality of $G$. This proves the assertion (1).

Assume that $N \notin Z(G)$. Then there exists a Sylow $t$-subgroup $S$ of $N$ such that $S \notin Z(G)$, since $N$ is nilpotent. Set $V=S / \Phi(S)$, where $\Phi(S)$ is the Frattini subgroup of $S$. Clearly $G$ acts on $V$. Let $R$ be the kernel of the action of $G$ on $V$. If $U$ is the Sylow $u$-subgroup of $N$, where $u$ is a prime, $u \neq t$, then $[S, U]=\langle 1\rangle$, since $N$ is nilpotent. This yields $N \unlhd R \unlhd G$, since $S^{\prime} \leq \Phi(S), S$ being a $t$-group. If $R=G$, then each Sylow $r$-subgroup of $G$, with $r \neq t$, centralizes $S$ by [22, Theorem 5.1.4]. That is $C_{G}(S) \not \leq N$. Furthermore, $C_{G}(S) \triangleleft G$ as $S \triangleleft G$. Then $N \triangleleft C_{G}(S) N \unlhd G$. Hence $G=C_{G}(S) N$, since $\bar{G}$ is non abelian and simple and $C_{G}(S) \not \leq N$. Actually, $G=C_{G}(S)$ since $N=\Phi(G)$ by Lemma 4.4. This gives a contradiction, since $S \notin Z(G)$. Hence $R<G$. Then $R=N$ as $\bar{G}$ is non abelian simple. Then $\bar{G} \leq \Gamma L(V)$, since $V$ is a vector space over $G F(t)$. Actually $\bar{G} \leq S L(V)$, since $\bar{G}$ is non abelian and simple. In particular, $G$ acts non trivially on the points of $P G(V)$ and hence $|V| \geq 1+d_{0}(\bar{G})$. This proves the assertion (2).

We point out that the condition in (2) of Lemma 5.1, in conjunction with the information contained in the paragraphs 5.3 and 5.4 of [43], furnishes a lower bound for $V$ and hence for $N$. This lower bound is generally greater than $1+d_{0}(\bar{G})$.

LEMMA 5.2. Let $\Omega$ be a set of nontrivial $N$-orbits of points (respectively lines) of $\Pi$ having the same length. If $G$ leaves $\Omega$ invariant then $|\Omega|=\sum_{j \geq 0} \lambda_{j} d_{j}(\bar{G})$, where $\lambda_{j} \geq 0$ for $j \geq 0$, and $\sum_{j \geq 0} \lambda_{j}>0$. In particular $|\Omega| \geq d_{0}(\bar{G})$.

Proof. Assume that $G$ fixes an element in $\Omega$. Then $G=G_{X} N$ for some point $X$ of $\Pi$ such that $X^{N} \in \Omega$. Actually $G=G_{X}$, since $N=\Phi(G)$ by Lemma 4.4. This yields $\left|X^{N}\right|=1$. This gives a contradiction, since $X^{N} \in \Omega$ and $\Omega$ is a set of non trivial $N$-orbits of points of $\Pi$. Thus $G$ acts on $\Omega$ as $\bar{G}$ and this moves each element of $\Omega$. Therefore $\Omega$ is union of non trivial $\bar{G}$-orbits. Since each non trivial $\bar{G}$-orbit has length a multiple of some primitive permutation representation degree $d_{h}(\bar{G})$ of $\bar{G}$, $h \geq 0$, we have that $|\Omega|=\sum_{j \geq 0} \lambda_{j} d_{j}(\bar{G})$, where $\lambda_{j} \geq 0$ for $j \geq 0$, and $\sum_{j \geq 0} \lambda_{j}>0$. In particular $|\Omega| \geq d_{0}(\bar{G})$.

\section{LEMMA 5.3. $\vec{G} ¥ P \Gamma L(2,8)$.}

Proof. Assume that $\bar{G} \cong P \Gamma L(2,8)$. Clearly $28<n \leq 56$. Set $L$ be the minimal preimage of $P S L(2,8)$ in $G$ and set $H=L \cap N$. Assume that $H=\langle 1\rangle$. Then $L \cong P S L(2,8)$. It is known that any involution $\zeta$ in $L$ fixes exactly four points on $\mathscr{O}$, since $|\mathscr{O}|=28$. Then $\zeta$ is a Baer collineation of $\Pi$. Thus either $n=36$ or $n=49$, since $n$ must be square and $28<n \leq 56$. The former is ruled out by [33, Theorem 3.6], since $o(\operatorname{Fix}(\zeta))=6$, and the latter is ruled out by [30]. Hence, we 
may assume that $H \neq\langle 1\rangle$. Then $H=\Phi(L)$ by the argument of Lemma 4.4 with $H$ in the role of $N$, since $L / H \cong P S L(2,8)$ is primitive on $\mathscr{O}$ as $|\mathscr{O}|=28$.

Now assume that $H \not Z Z(L)$. Note that the assertion of Lemma 5.1 is still true if we replace $\bar{G}$ with $L / H$, since the 2-transitivity is actually not required in that lemma. Thus there exists a Sylow $t$-subgroup $S$ of $H$ such that $P S L(2,8) \leq P \Gamma L(V)$, where $V=S / \Phi(S)$, since $L / H \cong P S L(2,8)$. Then either $|V| \geq 3^{7}$ or $8^{2}|| V \mid$ by $[43$, Theorem 5.3.9 and Proposition 5.4.13 respectively]. Therefore either $|H| \geq 3^{7}$ or $8^{2}|| H \mid$, since $V=S / \Phi(S)$. Then $|H|>n$ in any case, since $n \leq 56$. Thus $n$ and $|H|$ are powers of the same prime, $n|| H \mid$ and $|H| \mid n^{2}$ by Lemma 4.5 with $H$ in the role of $N$, since $H$ is transitive on $\mathscr{O}$. This rules out the case $|H| \geq 3^{7}$, since $n \leq 56$. Then $8^{2}|| H \mid$ and hence $n=2^{5}$, since $28<n \leq 56$. Let $\Omega$ be the set of $H$-orbits on $\Pi-l$. Then $|\Omega|=2^{4} / \theta$, since $n=2^{5},|H|=8^{2} \theta$, with $\theta$ a power of 2 , and since $H$ is semiregular on $\Pi-l$. On the other hand, $|\Omega|=\lambda_{0} 9$, with $\lambda_{0} \geq 0$, by Lemma 5.2, with $L$ in the role of $G$ and $H$ in the role of $N$, since 9 is the unique primitive permutation representation degree of $L / H$ less than 16. This gives a contradiction. Hence $L$ is a covering group for $P S L(2,8)$ by Lemma 5.1. Then $L \cong P S L(2,8)$, since the Schur multiplier of $\operatorname{PSL}(2,8)$ is trivial by $[43$, Theorem 5.1.4]. This gives a contradiction by the above argument.

Since $\bar{G} \not P \Gamma L(2,8)$, it follows, by a direct inspection of the list given in Theorem 2.1, that $\operatorname{soc}(\bar{G})$ is 2-transitive on $\mathscr{O}$. Thus we may assume that $\bar{G}=\operatorname{soc}(\bar{G})$. Hence $\bar{G}$ is a 2 -transitive non abelian simple group.

Let $K$ be the kernel of the action of $G$ on $l-\mathscr{O}$. Clearly $K \unlhd G$. Since $K N / N \unlhd \bar{G}$ and since $\bar{G}$ is non abelian and simple, either $G=K N$ or $K \leq N$. Actually $G=K$ in the first case, since $N=\Phi(G)$. So either $G=K$ and $G$ fixes $l-\mathscr{O}$ pointwise, or $K \leq N$. We now investigate the relationship between $N$ and $K$.

LEMMA 5.4. If $\bar{G} ¥ P S U(3,5)$ then either $N=N(l, l)$ or $N=N(Q, l)$ for some point $Q \in \Pi-l$. In particular $N \leq K$.

Proof. Assume that $N \notin K$. Then there exists a point $P \in l-\mathscr{O}$ such that $\left|P^{N}\right|>1$. Let $\Omega$ be the set of $N$-orbits on $P^{G}$. Then $|\Omega| \geq d_{0}(\bar{G})$ by Lemma 5.2, since $N \triangleleft G$ and $\left|P^{N}\right|>1$. Hence $\left|P^{N}\right| d_{0}(\bar{G}) \leq\left|P^{G}\right|$, since $|\Omega|=\left|P^{G}\right| /\left|P^{N}\right|$. This yields $2 d_{0}(\bar{G}) \leq v+1$, since $\left|P^{N}\right| \geq 2, P^{G} \subset l-\mathscr{O}$ and $|l-\mathscr{O}| \leq v+1$. Then $d_{0}(\bar{G})<v$. In particular $\left|P^{N}\right|=2$ and either $\bar{G} \cong P S L(4,2)$ or $\bar{G} \cong A_{7}$, by Lemma 4.3 (2), since $\bar{G} ¥ P S U(3,5)$ by our assumption.

Assume that $\bar{G} \cong P S L(4,2)$ or $\bar{G} \cong A_{7}$. Note that $n+1 \geq v+2 d_{0}(\bar{G})$ in any of these cases. Thus either $n=30$ and $\bar{G} \cong P S L(4,2)$ or $n \in\{28,29,30\}$ and $\bar{G} \cong A_{7}$, since $n \leq 2 v$. The case $n=30$ is ruled out by [33, Theorem 13.18]. Hence $\bar{G} \cong A_{7}$ and $n \in\{28,29\}$. Assume that 2||$N \mid$ and $2^{2} \nmid|N|$. Then $N$ contains a Baer collineation of $\Pi$, since $N$ fixes $\mathscr{O}$ pointwise and $\left|P^{N}\right|=2$. This gives a contradiction, 
so $2^{2}|| N \mid$. Let $L$ be a Sylow 2-subgroup of $G$. Then $2^{5}|| L \mid$, since $2^{3}|| A_{7} \mid$ and $2^{2}|| N \mid$. It is easily seen that there exists a non trivial subgroup $L_{0}$ of $L$ fixing at least two points on $\Pi-l$, since $n^{2} \not \equiv 0,1 \bmod 2^{5}$ as $n \in\{28,29\}$. Then $L_{0} \cap N=\langle 1\rangle$, since $N$ fixes at most one point $\Pi-l$ by Lemma 4.5. Then $L_{0}$ and hence $G$ contain an involution $\xi$ acting faithfully on $\mathscr{O}$. In particular $\zeta$ is a Baer collineation of $\Pi$, since $\zeta$ fixes at least three points on $\mathscr{O}$ by [50]. So $n$ must be a square. This gives a contradiction, so $N \leq K$ and the assertion follows by Lemma 4.5 .

At this point we study the cases when either $N=N(l, l)$ or $N=N(Q, l)$ for some point $Q \in \Pi-l$, for $d_{0}(\bar{G})=v$ and $d_{0}(\bar{G})<v$, separately.

5.1.1. The case $d_{0}(\bar{G})=v \quad$ In this subsection, under the assumption $d_{0}(\bar{G})=v$, we prove that for $N=N(l, l)$ or $N=N(Q, l)$, where $Q \in \Pi-l$, the group $G$ is a perfect central extension of $\bar{G}$ and that each involution of $G$ lies in $N$. From this we deduce that the Sylow 2-subgroups of $G$ are dihedral and then we use the Gorenstein-Walter Theorem [23] to complete our treatment of this case.

PROPOSITION 5.5. If $N=N(Q, l)$ where $Q \in \Pi-l$, then $N=K$. Furthermore, the following occur:

(A) $n=2 v-1$ and $G$ acts on $l-\mathscr{O}$ as $\bar{G}$ in its 2-transitive permutation representation of degree $v$;

(B) $G$ is a covering group for $\bar{G}$;

(C) each involution of $G$ lies in $N$.

PROOF. We proceed in a series of steps.

(A) $G$ acts on $l-\mathscr{O}$ as $\bar{G}$ in its 2-transitive permutation representation of degree $v$. In particular $N=K$ and $n=2 v-1$.

Assume that $G$ fixes a point $A$ on $l-\mathscr{O}$. Denote by $\Sigma$ the set of $N$-orbits of points of $A Q-\{A, Q\}$. Then $|\Sigma|=(n-1) /|N|$, since $N$ is semiregular on $A Q-\{A, Q\}$. In particular, $|\Sigma| \geq d_{0}(\bar{G})$ by Lemma 5.2 , since $G$ acts on $\Sigma$. This yields $d_{0}(\bar{G})|N| \leq n-1$, since $|\Sigma|=(n-1) /|N|$. This gives a contradiction, since $d_{0}(\bar{G})=v, n \leq 2 v$ and $|N| \geq 2$. Thus $G$ fixes no points on $l-\mathscr{O}$. Hence $N=K$, where $K$ is the kernel of the action of $G$ on $l-\mathscr{O}$, by Lemma 5.4. Moreover, $G$ acts on $l-\mathscr{O}$ as $\bar{G}$ and $\bar{G}$ fixes no points on $l-\mathscr{O}$. This yields $n+1-v=\sum_{j \geq 0} \lambda_{j} d_{j}(\bar{G})$, where the $\lambda_{j} \geq 0, j \geq 0$, and $\sum_{j \geq 0} \lambda_{j}>0$, since each $\bar{G}$-orbit on $l-\mathscr{O}$ is a multiple of some $d_{h}(\bar{G}), h \geq 0$, and since $|l-\mathscr{O}|=n+1-v$. Actually, either $\lambda_{0}=1$ and $\lambda_{j}=0$ for $j>0$, or there exists $\bar{j}>0$, such that $\lambda_{\bar{j}}=1, d_{\bar{j}}(\bar{G})=v+1$ and $\lambda_{j}=0$ for each $j \geq 0$ such that $j \neq \bar{j}$, since $d_{0}(\bar{G})=v$.

If the latter occurs then $n=2 v$. Furthermore, $\bar{G}$ is one of the exceptions listed in Lemma 1.1. Nevertheless, no one of these exceptions really occurs, since $v$ must be 
even by [33, Theorem 13.18]. Hence $\lambda_{0}=1$ and $\lambda_{j}=0$ for $j>0$ for any admissible case. Then $n=2 v-1$ and hence $G$ acts on $l-\mathscr{O}$ as $\bar{G}$ in its 2-transitive permutation representation of degree $v$.

\section{(B) $\boldsymbol{G}$ is a covering group for $\overline{\boldsymbol{G}}$.}

Assume that $N \not Z Z(G)$. Then there exists a Sylow $t$-subgroup $S$ of $N$ such that $|S| \geq 1+v$ by Lemma 5.1 , since $d_{0}(\bar{G})=v$. Furthermore, either $2|S| \leq n-1$ or $|S|=n-1$ since $S \leq N$ and $|N| \mid n-1$. The former is ruled out, since $2(v+1) \leq n-1$ as $|S| \geq 1+v$ and $n \leq 2 v$. Hence $S=N$ and $|S|=n-1$. Thus $n-1=t^{k}, k \geq 1$, since $S$ is a $t$-group. This yields $t=2$ and hence $v=2^{k-1}+1$, since $n=2 v-1$.

Assume that $G$ contains a Baer collineation of $\Pi$. Thus $n$ must be a square. Then $n=9$ and $k=3$ by [54, Result A5.1], since $n-1=t^{k}$. This gives a contradiction by [30, Theorem A]. Hence $G$ contains no Baer collineations of $\Pi$.

Now let $S$ be any Sylow 2-subgroup of $G$. Then $S$ fixes a point $C$ on $\mathscr{O}$, since $v$ is odd and $v>2$. Thus $S=S_{B} N$ and $S_{B} \cap N=\langle 1\rangle$ for some point $B \in Q C-\{Q, C\}$, since $N$ is regular on $Q C-\{Q, C\}$ because $N=N(Q, l)$ and $|N|=n-1$. In particular, each involution in $S_{B}$ is a homology of $\Pi$ with centre lying on $l$ and axis distinct from $l$, since $G$ fixes $l, G$ contains no Baer collineations of $\Pi, n$ is odd and $S_{B} \cap N=\langle 1\rangle$. Moreover, any involution in $S_{B}$ commutes with some involution in $N$ since $N \leq S$. Then $N$ contains exactly one involution by [39, Lemma 2.1 (ii)], since $N=N(Q, l)$. Actually, the previous argument shows that there exists at most one involutory homology in $G$ with given centre and axis. Then $\bar{G} \cong P S L(2, q), q$ odd, by [14, Theorem 1], since $\bar{G}$ is non-abelian and simple. Then $v=q+1, q$ odd, since $d_{0}(\bar{G})=v$. This gives a contradiction, since $v=2^{k-1}+1$.

\section{(C) Each involution of $G$ lies in $N$.}

Suppose that there exists an involution $\sigma \in G-N$. Assume that $\sigma$ is a Baer collineation of $\Pi$. Then $\sqrt{n}+1=2 k_{\sigma}$, where $k_{\sigma}$ denotes the number of points of $\Pi$ fixed by $\sigma$ on $\mathscr{O}$ and on $l-\mathscr{O}$, since $G$ acts on $\mathscr{O}$ and on $l-\mathscr{O}$ as $\bar{G}$ in the same way by (A). Hence $k_{\sigma}=(\sqrt{2 v-1}+1) / 2$. Note that $v$ is known and $k_{\sigma}$ can easily be recovered from the structure of $\bar{G}$ and the action of $\bar{G}$ on $\mathscr{O}$ for each 2-transitive non abelian simple group $\bar{G}$ listed in Theorem 2.1. Hence, we may filter the list given in Theorem 2.1 with respect to $k_{\sigma}=(\sqrt{2 v-1}+1) / 2$. So it remains to investigate the following admissible cases:

(i) $\bar{G} \cong A_{v}, v \geq 5$;

(ii) $\bar{G} \cong P S L(d, q), d \geq 2, q=p^{r},(d, q) \neq(2,2),(2,3)$.

Assume that $\bar{G} \cong A_{v}, v \geq 5$. Note that $v \notin\{6,7\}$, since $n=2 v-1$ must be a square. Thus $N \cong Z_{2}$ by (B) and by [43, Theorem 5.1.4], since $N \neq\langle 1\rangle$. Let $Y \in \mathscr{O}$ and denote by $\Gamma$ the set of $N$-orbits on $Q Y-\{Q, Y\}$. Then $|\Gamma|=(n-1) /|N|$, 
since $N$ is semiregular on $Q Y-\{Q, Y\}$. Furthermore $G_{Y}$ acts on $\Gamma$, since $N \triangleleft G$. In particular $\bar{G}_{Y} \cong A_{v-1}$. Assume that $G_{Y}$ fixes an element on $\Gamma$. Then $G_{Y}=G_{Y, O} N$ and $G_{Y, O} \cap N=\langle 1\rangle$ for some point $O \in Q Y-\{Q, Y\}$, since $N$ is semiregular on $Q Y-\{Q, Y\}$. In particular $G_{Y, O}$ acts on $l-\{Y\}$ as $\bar{G}_{Y}$. Now, pick a 3-cycle $\zeta$ in $G_{Y, O}$. Clearly $\zeta$ fixes $v-3$ points on $\mathscr{O}$, since $G_{Y, O}$ acts on $l-\{Y\}$ as $\bar{G}_{Y}$. Then $\zeta$ fixes $v-3$ points on $l-\mathscr{O}$, since the action of $G$ on $\mathscr{O}$ and on $l-\mathscr{O}$ is the same. So, $\zeta$ fixes exactly $n-6$ points on $l$, since $n=2 v-1$. Furthermore, $\zeta$ fixes the points $Q$ and $O$, with $O, Q \in \Pi-l$, since $\zeta$ lies in $G_{Y, o}$. Therefore, $\zeta$ fixes a subplane of $\Pi$ of order $n-7$. Then $(n-7)^{2} \leq n$ by [33, Theorem 3.7]. This yields either $n=9$ or $n=10$, since $n \geq 9$ by our assumption. Nevertheless, these cases cannot occur by [30, Theorem A] and [33, Theorem 13.18$]$ respectively. As a consequence, $G_{Y}$ moves each element on $\Gamma$. Therefore $\Gamma$ is a union of non trivial $\bar{G}_{Y}$-orbits. Since each $\bar{G}_{Y}$-orbit on $\Gamma$ is a multiple of some $d_{j}\left(A_{v-1}\right), j \geq 0$, we have $\sum_{j \geq 0} \lambda_{j} d_{j}\left(A_{v-1}\right)=|\Gamma|$. That is $2 \sum_{j \geq 0} \lambda_{j} d_{j}\left(A_{v-1}\right)=n-1$, since $|\Gamma|=(n-1) /|N|$ and $N \cong Z_{2}$. Then $\lambda_{0}=1$ and $\lambda_{j}=0$ for $j>1$, since $d_{o}\left(A_{v-1}\right)=v-1$ and $n=2 v-1$. Hence $\bar{G}_{Y} \cong A_{v-1}$ acts in its 2 -transitive permutation representation of degree $v-1$ on $\Gamma$. Then there still exists a 3 -cycle $\zeta$ in $G$ fixing $n-6$ points on $l$ and at least 2 points on $Q Y-\{Y\}$. Hence $\zeta$ fixes a subplane of $\Pi$ of order $n-7$. Again, this gives a contradiction.

Assume that $\bar{G} \cong P S L(d, q), d \geq 2, q=p^{r},(d, q) \neq(2,2),(2,3)$. Note that $(d, q) \neq(2,5),(2,9)$, since the cases $\operatorname{PSL}(2,5) \cong A_{5}$ and $v=5, \operatorname{PSL}(2,9) \cong A_{6}$ and $v=6$ have been ruled out above. Also the case $(d, q)=(2,7)$ and $v=7$, or $(d, q)=(2,11)$ and $v=11$ are ruled out since in these cases $n$ is a nonsquare. Thus $d_{0}(\bar{G})=\left(q^{d}-1\right) /(q-1)$ by [12]. Hence $n=2\left[\left(q^{d}-1\right) /(q-1)\right]-1$. Let $E$ be an elementary abelian subgroup of $G$ of order $q^{d-1}$ which induces on $\mathscr{O}$ a group of projective transvections with the same fixed hyperplane. Let $B$ be a point on $\mathscr{O}$ fixed by $E$. If there exists a nontrivial element $\delta$ in $E$ fixing a point on $Q B-\{Q, B\}$, then $\delta$ is planar on $\Pi$. In particular $\delta$ fixes exactly $2\left(q^{d-1}-1\right) /(q-1)$ points on $l$, since the action of $G$ on $\mathscr{O}$ and $l-\mathscr{O}$ is the same, and since $\delta$ fixes exactly $\left(q^{d-1}-1\right) /(q-1)$ points on $\mathscr{O}$. So $\left(2\left[\left(q^{d-1}-1\right) /(q-1)\right]-1\right)^{2} \leq 2\left[\left(q^{d}-1\right) /(q-1)\right]-1$ by $[33$, Theorem 3.7]. Thus $d=2$ and hence $G \cong S L(2, q)$ by [41, Theorem 7.1.1(i)], since $q \notin\{5,7,9,11\}$. This gives a contradiction, since the unique involution of $G \cong S L(2, q)$ lies in $N$. Hence $E$ is semiregular on $Q B-\{Q, B\}$. Thus $q^{d-1} \mid n-1$. Then either $d=2$ or $(d, q)=(3,2)$, since $n=2\left[\left(q^{d}-1\right) /(q-1)\right]-1$. Again, this gives a contradiction.

The above argument leads us to assert that each involution in $G-N$ must be a homology of $\Pi$ as $n$ is odd. Assume that $N$ has even order. Any involution in $G$ commutes with $N$ as $G$ is a covering group for $\bar{G}$ by (B). Thus $N$ contains exactly one involution by [39, Lemma 2.1 (ii)], since $N=N(Q, l), N$ has even order and since $G-N$ contains involutions. Actually, the previous argument yields shows that 
there exists at most one involutory homology in $G$ with given centre and axis. Then $G \cong S L(2, q), q$ odd, by Theorem 1 of [14] and by [41, Theorem 7.1.1(i)], since $G$ is a covering group for $\bar{G}$ and since $q \notin\{5,7,9\}$. This gives a contradiction, since there are no involutions in $G-N$. Hence $N$ has odd order. Let $\alpha$ be any involution of $G-N$. Then $\alpha$ fixes exactly 2 points on $l$, since $\alpha$ is a homology and $\alpha$ fixes $l$. In particular, $\alpha$ fixes exactly one point on $\mathscr{O}$ and one on $l-\mathscr{O}$ since the action of $G$ on $\mathscr{O}$ and $l-\mathscr{O}$ is the same. Thus $G_{D} / N$ has even order for any $D \in \mathscr{O}$. Moreover, $G_{D_{1}, D_{2}} / N$ has odd order for any two distinct points $D_{1}$ and $D_{2}$ of $\mathscr{O}$, since $N$ has odd order and each involution in $G-N$ is a homology of $\Pi$. Then $\bar{G} \cong P S L\left(2,2^{s}\right)$ or $\bar{G} \cong S z\left(2^{s}\right)$ or $\bar{G} \cong P S U\left(3,2^{s}\right)$ by [3]. Actually, $G \cong S L(2,5)$ or $\bar{G} \cong S z(8)$ or $\bar{G} \cong P S U\left(3,2^{s}\right)$ and $N \cong Z_{3}$ by [43, Theorem 5.1.4], as $N \neq\langle 1\rangle$. Nevertheless, the case $G \cong S L(2,5)$ cannot occur, since $N \cong Z_{2}$ and any Sylow 2-subgroup of $G$ is isomorphic to $Q_{8}$. The case $\bar{G} \cong S z(8)$ cannot occur by [33, Theorem 3.6] since $n=129$. Finally, the case $\bar{G} \cong \operatorname{PSU}\left(3,2^{s}\right)$ cannot occur, since $n=2^{3 s+1}+1$ while $|N|=3$ must be a divisor of $n-1$.

PROPOSITION 5.6. If $N=N(l, l)$, then $N=K$. Furthermore, the following occur:

(A) $G$ fixes exactly one point $X$ on $l-\mathscr{O}$;

(B) $n=2 v$ and $G$ acts on $l-(\mathscr{O} \cup\{X\})$ as $\bar{G}$ in its 2-transitive permutation representation of degree $v$;

(C) $N=N(X, l)$;

(D) each involution of $G$ lies in $N$;

(E) $G$ is a covering group for $\bar{G}$.

Proof. Assume that $N=N(l, l)$. We proceed in a series of steps.

\section{(A) $G$ fixes at least a point $X$ on $l-\mathscr{O}$.}

Assume that $\bar{G}$ fixes no points on $l-\mathscr{O}$. Then $l-\mathscr{O}$ is union of nontrivial $\bar{G}$-orbits. This yields $n+1-v=\sum_{j \geq 0} \theta_{j} d_{j}(\bar{G})$, where $\theta_{j} \geq 0, j \geq 0$, and $\sum_{j \geq 0} \theta_{j}>0$, since each $\bar{G}$-orbit on $l-\mathscr{O}$ is a multiple of some $d_{h}(\bar{G}), h \geq 0$, and since $|l-\mathscr{O}|=n+1-v$. At this point we may use the same argument as in part (A) of Proposition 5.5 to show that $n=2 v-1$ and $G$ acts on $l-\mathscr{O}$ as $\bar{G}$ in its 2-transitive permutation representation of degree $v$. Set $|N(l, l)|=p^{h}$ with $h>0,|N(C, l)|=p^{i}$ with $i \geq 0$, for any $C \in \mathscr{O}$, and set $|N(D, l)|=p^{j}, j \geq 0$, for any $D \in l-\mathscr{O}$. Clearly $i+j>0$, since $N \neq\langle 1\rangle$. Furthermore $h \geq i, j$. Then

$$
\left(p^{i}-1\right) \frac{(n+1)}{2}+\left(p^{j}-1\right) \frac{(n+1)}{2}+1=p^{h},
$$

since $\bar{G}$ has the same 2-transitive permutation representation on $\mathscr{O}$ and $l-\mathscr{O}$, and since $v=(n+1) / 2$. By manipulating (5.1), we have that

$$
\left(p^{i}+p^{j}\right)(n+1)=2\left(p^{h}+n\right) \text {. }
$$


As $p \mid n$ and $h>0$, then $p \mid\left(p^{i}+p^{j}\right)(n+1)$ and hence $i, j>0$. Furthermore $p^{i} \mid n$ and $p^{j} \mid n$, since $N(C, l)$ is semiregular on $[D]-\{l\}$ and $N(D, l)$ is semiregular on $[C]-\{l\}$. Thus $p^{f} \mid n$, where $f=\max \{i, j\}$. Then $p^{f} \mid\left(p^{i}+p^{j}\right)$, since $p^{f} \mid\left(p^{h}+n\right)$. Hence $i=j$, with $i, j>0$. Then $\Pi$ is a translation plane and $N$ is regular on $\Pi-l$ by [33, Theorem 4.26]. Therefore $G=N G_{o}$ for some point $O \in \Pi-l$. Then $G=G_{o}$, since $N=\Phi(G)$ by Lemma 4.4. So $N$ fixes $O$, which is a contradiction, since $N$ is semiregular on $\Pi-l$. Thus $N$, and hence $G$, fix at least a point $X$ on $l-\mathscr{O}$.

(B) $G$ acts on $l-(\mathscr{O} \cup\{X\})$ as $\bar{G}$ in its 2-transitive permutation representation of degree $v$. In particular $N=K$ and $n=2 v, v$ even.

Suppose that $G$ fixes $l-\mathscr{O}$ pointwise. Assume that there exists a point $P$ of $l-\mathscr{O}$ such that $N(P, l)=\langle 1\rangle$. Let $\Lambda$ be the set of the $N$-orbits on $[P]-\{l\}$. By Lemma 5.2, we have that $|\Lambda| \geq d_{0}(\bar{G})$. By an argument similar to that used in (A) of Proposition 5.5 we deduce that $n=2 v$ and $N \cong Z_{2}$, since $|\Lambda|=n /|N|, d_{0}(\bar{G})=v$ and $v<n \leq 2 v$. Thus $N \leq Z(G)$. Let $x \in G, x \neq 1$, be such that $o(x) \mid v-1$. Note that $v-1$ is odd by [33, Theorem 13.18], since $n=2 v$. Then $x$ fixes $v+1$ points on $l$, since $G$ fixes $l-\mathscr{O}$ pointwise and $|l-\mathscr{O}|=v+1$. Furthermore, $x$ fixes a point $R$ on $\Pi-l$, since $n=2 v$. Let $\sigma \in N$. Then $R \sigma \in \operatorname{Fix}(x)$, since $N \leq Z(G)$. Note that $R \sigma \neq R$, since $\sigma \in N, R \in \Pi-l$ and $N=N(l, l)$. Thus $x$ is planar on $\Pi$ and $o(\operatorname{Fix}(x)) \geq v$. Then $v^{2} \leq n \leq 2 v$ by [33, Theorem 3.7]. This gives a contradiction, since $v>2$.

Assume that $N(B, l) \neq\langle 1\rangle$ for each $B \in l-\mathscr{O}$. Let $Y$ be any point of $l-\mathscr{O}$. Let $\Gamma$ be the set of the $N$-orbits on $[Y]-\{l\}$. Then $|\Gamma|=n /[N: N(Y, l)]$, since $N(Y, l)<N$ as $N(B, l) \neq\langle 1\rangle$ for each $B \in l-\mathscr{O}$. A similar argument to that used above yields $n=2 v$ and $[N: N(Y, l)]=2$ for any $Y \in l-\mathscr{O}$, since $d_{0}(\bar{G})=v$ and $v<n \leq 2 v$. Thus $N \cong E_{4}$ and hence $|l-\mathscr{O}|=3$. That is $n+1-v=3$, which is a contradiction, since $n=2 v$ and $v \geq 5$.

(C) $N=N(X, l)$.

Assume that $N(X, l)<N$. We may repeat the previous argument on the set of the $N$-orbits on $[X]-\{l\}$ to show that $[N: N(X, l)]=2$. Hence $N$ is an elementary abelian 2-group, since $N=N(l, l)$ and $N(X, l)<N$. Set $|N(X, l)|=2^{f}, f \geq 0$, and set $|N(C, l)|=2^{i}, i \geq 0$, for any $C \in \mathscr{O}$. Set also $|N(D, l)|=2^{j}, j \geq 0$, for any $D \in l-(\mathscr{O} \cup\{X\})$ by $(\mathbf{B})$. Then

$$
\left(2^{i}-1\right) v+\left(2^{j}-1\right) v+2^{f}=2^{f+1} .
$$

Furthermore $(i, j)=(1,1),(1,0)$ or $(0,1)$, since $[N: N(X, l)]=2$. In the case where $(i, j)=(1,1), f=1$ and $v=1$ by [33, Theorem 4.26], which gives a contradiction. Hence, either $(i, j)=(1,0)$ or $(0,1)$. It is easily seen that $v=2^{f}$ and $n=2^{f+1}$ in any of these two cases. In particular $|N(X, l)|=n / 2$. We may assume that $(i, j)=(0,1)$, since the role of $i$ and $j$ can be exchanged in the following argument. 
Note that $N(X, l) \triangleleft G$, since $G$ fixes $X$. Assume that $N(X, l) \not Z Z(G)$. Then $|N(X, l)| \geq 1+v$, since $G$ acts on $N(X, l)$ as $\bar{G}$ since $N$ is abelian and $d_{0}(\bar{G})=v$. This gives a contradiction, since $|N(X, l)|=2^{f}=v$. Hence $N(X, l) \leq Z(G)$. Let $\zeta$ be an element of prime order dividing $v-1$. Then $\zeta$ must a 2-element by [22, Corollary 5.3.3], since $N / N(X, l) \cong Z_{2}$ and $N(X, l) \leq Z(G)$. This is a contradiction, since $v-1$ is odd. Hence $N=N(X, l)$.

\section{(D) Each involution of $G$ lies in $N$.}

Suppose that there exists an involution $\sigma \in G-N$. Assume that $\sigma$ is a $\left(C_{\sigma}, l_{\sigma}\right)$ elation of $\Pi$. If $C_{\sigma}=X$, then $N<G(X, X) \triangleleft G$. This gives a contradiction, since $\bar{G}$ is non-abelian and simple. Hence $C_{\sigma} \neq X$. Furthermore $a_{\sigma} \neq l$, since $\sigma \notin N$. Denote by $R$ the normal closure of $\langle\sigma\rangle$ in $G$. Then $G=R N$, since $\bar{G}$ is non-abelian and simple. Actually, $G=R$ by the minimality of $G$. Hence $G$ is generated by involutory elations. Moreover $N=F(G)$, where $F(G)$ denotes the Fitting subgroup of $G$, since $N$ is nilpotent and $\bar{G}$ is non-abelian and simple. Since 4|| $\bar{G} \mid$ by [21], it follows that $\bar{G}$ is isomorphic to $\operatorname{PSL}(3, q)$ or $\operatorname{PSU}(3, q)$ or $S L(2, q)$ or $S z(q)$ or $A_{6}$, where $q=2^{r}$, by [27]. This is a contradiction in all cases except $A_{6}$, since $v$ must be even by [33, Theorem 13.18]. Nevertheless, the case $\bar{G} \cong A_{6}$ is ruled out by [36], since $n=12$.

Assume that $\sigma$ is a Baer collineation of $\Pi$. Then $\sqrt{n}+1=2 k_{\sigma}+1$, where $k_{\sigma}$ is the number of points of $\Pi$ fixed by $\sigma$ both on $\mathscr{O}$ and on $l-(\mathscr{O} \cup\{X\})$. Hence $k_{\sigma}=\sqrt{v / 2}$. Now, arguing similarly to part $(\mathbf{C})$ of Proposition 5.5, we may reduce our investigation to the following admissible cases:

(i) $\bar{G} \cong A_{v}, v \geq 5$;

(ii) $\bar{G} \cong P S L(d, q), d \geq 2, q=p^{r},(d, q) \neq(2,2),(2,3)$.

Assume that $G \cong A_{v}, v \geq 5$. Let $Y \in \mathscr{O}$ and denote by $\Psi$ the set of $N$-orbits on $[Y]-\{l\}$. Then $|\Psi|=n /|N|$ as $N$ is semiregular on $[Y]-\{l\}$, since $N=N(X, l)$ with $X \neq Y$. Furthermore, $G_{Y}$ acts on $\Psi$, since $N \triangleleft G$. In particular $\bar{G}_{Y} \cong A_{v-1}$. Assume that $G_{Y}$ fixes an element on $\Psi$. Then $G_{Y}=G_{Y, r} N$ and $G_{Y, r} \cap N=\langle 1\rangle$ for some point $r \in[Y]-\{l\}$. So $\sigma$ may be picked in $G_{Y, r}$ as a product of two transpositions on $\mathscr{O}$. Then $\sigma$ fixes exactly $n-7$ points on $l$, other than $X$, since $\bar{G}$ acts on $\mathscr{O}$ and on $l-(\mathscr{O} \cup\{X\})$ in the same way. Then $(n-7)^{2}=n$ by [33, Theorem 3.7]. This gives a contradiction. As a consequence, $G_{Y}$ moves each element on $\Psi$ and $\Psi$ is a union of non trivial $\bar{G}_{Y}$-orbits. Since each $\bar{G}_{Y}$-orbit on $\Psi$ is a multiple of some $d_{j}\left(A_{v-1}\right), j \geq 0$, then $\sum_{j \geq 0} \lambda_{j} d_{j}\left(A_{v-1}\right)=|\Psi|$. That is $|N| \sum_{j \geq 0} \lambda_{j} d_{j}\left(A_{v-1}\right)=2 v$ as $|\Psi|=n /|N|$ and $n=2 v$. This gives a contradiction for $v \neq 6,8$, by Lemma 4.2 applied to $\bar{G}_{Y} \cong A_{v-1}$ as $d_{0}\left(A_{v-1}\right)=v-1$ and $|N| \geq 2$. Actually, the case $v=6$ and $\bar{G}_{Y} \cong A_{5}$ cannot occur by [36], since $n=2 v$. Hence $\bar{G} \cong A_{8}$ and $n=16$. In this case $\sigma$ fixes 4 points on $\mathscr{O}$ and 4 points on $l-\mathscr{O}$. So $\sigma$ fixes at least 8 points on $l$ and $\sigma \notin N$, which is a contradiction by [33, Theorem 3.7]. 
Assume that $G \cong P S L(d, q), d \geq 2, q=p^{r},(d, q) \neq(2,2),(2,3)$. Note that $(d, q) \neq(2,5),(2,7),(2,11)$, since $v$ must be even by [33, Theorem 13.18]. Also, the case $(d, q)=(2,9)$ is ruled out, since $\operatorname{PSL}(2,9) \cong A_{6}$. Thus by [12] $d_{0}(\bar{G})=\left(q^{d}-1\right) /(q-1)$ and hence $n=2\left(q^{d}-1\right) /(q-1)$. Note that $q$ is odd and $d$ is even, since $v$ must be even. Moreover $n \not \equiv 0,1 \bmod p$. Thus a similar argument to that used in part (C) of Proposition 5.5 shows that $G$ always contains planar $p$ elements fixing $2\left(q^{d-1}-1\right) /(q-1)$ points on $l-\{X\}$, as $n \neq \equiv, 1 \bmod p$. This yields $d=2$ by [33, Theorem 3.7]. Hence $G \cong S L(2, q)$ by [41, Theorem 7.1.1.(i)], since $q \notin\{5,7,9,11\}$. This is a contradiction, since the unique involution of $G \cong S L(2, q)$ lies in $N$.

\section{(E) $G$ is a covering group for $\overrightarrow{\boldsymbol{G}}$.}

Assume that $N \not Z Z(G)$. Then there exists a Sylow $t$-subgroup $S$ of $N$, such that $|S|>1+v$ by Lemma 5.1. On the other hand, $|S| \mid 2 v$ since $S \leq N,|N| \mid n$ by (C), and $n=2 v$ by (B). Thus $|S|=n$, since $|S|>1+v$. Hence $S=N$. Then $N$ is a 2-group and $n$ is a power of 2, since $n=2 v$ and $|N| \mid n^{2}$. Let $A \in \mathscr{O}$. Then $N(A, l)=\langle 1\rangle$ by Lemma 4.5 , since $|N|=n$. Thus $N$ is regular on $[A]-\{l\}$ and hence $G_{A}=G_{A, s} N$ and $G_{A, s} \cap N=\langle 1\rangle$ for some point $s \in[A]-\{l\}$. Furthermore $G_{A, s} \cong \bar{G}_{A}$. Then $G_{A, s}$ must have odd order, since each involution in $G$ actually lies in $N$ by (D) and since $N=N(X, l)$ with $X \neq A$. This implies that each involution in $\bar{G}$ fixes no points on $\mathscr{O}$. Then $\bar{G} \cong P S L(2, q)$ with $q \equiv 3 \bmod 4$, by [2]. By Lemma 5.1(2), we have that $P S L(2, q) \leq P S L(V)$, where $V=S / \Phi(S)$ and this implies $|S|>1+v$ as $d_{0}(\bar{G})=v$. Actually, in this case $|S| \geq 2^{(q-1) / 2}$ by $[43$, Theorem 5.3.9], since $q \equiv 3 \bmod 4$. Then $2^{(q-1) / 2} \leq 2(q+1)$, since $|S| \mid 2(q+1)$ as $S \leq N,|N| \mid n$ and $n=2(q+1)$ with $q \equiv 3 \bmod 4$. This gives a contradiction, since $q \neq 7,11$ as $q \equiv 3 \bmod 4$ and $d_{0}(\bar{G})=v$.

THEOREM 5.7. Let $\Pi$ be a projective plane of order $n$ and let $\mathscr{O}$ be a 2-transitive $G$-orbit of length $v$ on a line with $n>v \geq n / 2$. If $G$ is almost simple and $d_{0}(\bar{G})=v$ then one of the following occurs:

(1) $\Pi$ is the Hall plane of order 9 or its dual, $G \cong S L(2,5)$ and $|\mathscr{O}|=5$;

(2) $n=2 q+1, G \cong S L(2, q)$ with $q \equiv 3 \bmod 4, q \neq 7$, and $|\mathscr{O}|=q+1$;

(3) $n=2(q+1), G \cong S L(2, q)$ with $q \equiv 3 \bmod 4$, and $|\mathscr{O}|=q+1$.

Proof. Note that $N \cong Z_{2}$, unless $\bar{G} \cong P S L(3,4)$ or $\bar{G} \cong S z(8)$, by Proposition 5.5 (B) and (C), by Proposition 5.6 (D) and (E), and by [43, Theorem 5.1.4], since $d_{0}(\bar{G})=v$.

Assume that $\bar{G} \cong P S L(3,4)$ or $\bar{G} \cong S z(8)$ and assume that $N \not Z_{2}$. Note that the case $n=2 v$ cannot occur by [33, Theorem 13.18], since $v$ is odd in both cases. Then $n=2 v-1$ and $N=N(Q, l)$ for some point $Q \in \Pi-l$ fixed by $G$, by Proposition 5.6. Nevertheless, the case $\bar{G} \cong S z(8)$ cannot occur by [33, Theorem 3.6], since $n=129$ 
in this case. Hence $\bar{G} \cong P S L(3,4)$ and $n=41$. Let $U$ be a Sylow 2-subgroup of $G_{J}$, where $J$ is any point of $\mathscr{O}$. Then $U$ must be semiregular on $Q J-\{Q, J\}$, since each involution in $G$ lies in $N$ by Proposition 5.5(3). Hence $|U| \mid n-1$. This gives a contradiction, since $2^{6}|| U \mid$ and $n=41$.

Assume that $N \cong Z_{2}$ in any admissible case. Thus each Sylow 2-subgroup $S$ of $G$ is isomorphic either to $Z_{2^{m}}$ or to $Q_{2^{m}}$ for some positive integer $m$ in any case by Proposition 5.5(C) and Proposition 5.6(D). In the first case we have $S /(S \cap N) \cong Z_{2^{m-k}}$, where $0<k \leq m$. Nevertheless this case is ruled out by [21, Theorem 4] applied to $\bar{G}$, since this one is non abelian and simple. Hence $S \cong Q_{2^{m}}$ and hence $S /(S \cap N) \cong D_{2^{m-1}}$, since $S \cap N \cong Z_{2}$. By [23], either $\bar{G} \cong P S L(2, q)$ with $q$ odd, or $\bar{G} \cong A_{7}$. If $\bar{G} \cong A_{7}$ then $n=28$ or 29 , and this case cannot occur by the same argument as in Proposition 5.4. Hence, we may assume that $\bar{G} \cong P S L(2, q)$ with $q$ odd. Assume that $q=5$. Then $n=9$ or $n=10$, since $d_{0}(\bar{G})=5$. Actually, the latter is ruled out by $[33$, Theorem 13.18]. Hence $n=9$ and either $\Pi \cong P G(2,9)$ or $\Pi$ is the Hall plane of order 9 or $\Pi$ is the dual of the Hall plane of order 9 by [58]. This proves the assertion (1).

Assume that $q=7$. Then either $n=13$ or $n=14$ since $d_{0}(\bar{G})=7$. The latter is ruled out by [33, Theorem 13.18]. Hence $n=13$. Then $\Pi \cong P G(2,13)$ by [48], since $d_{0}(\bar{G})=7$. Hence $\bar{G} \leq P G L(2,13)$, since $G$ fixes $l$. This gives a contradiction.

Now assume that $q=9$. Then either $n=11$ or $n=12$, since $d_{0}(\bar{G})=9$. The latter is ruled out by [36]. Hence $n=11$ and $\Pi \cong P G(2,11)$ by [47]. Hence $\bar{G} \leq P G L(2,13)$, since $G$ fixes $l$. This gives a contradiction, hence $q \notin\{7,9\}$. Thus $G \cong S L(2, q)$ by [41, Theorem 7.1.1.(i)], for $q \neq 5$, as $q \notin\{7,9\}$.

Assume that $n=2 v-1$. Let $R$ be a Sylow 2-subgroup of $G_{B}$, where $B$ is any point of $\mathscr{O}$. Then $R$ must be semiregular on $Q B-\{Q, B\}$, since each involution in $G$ lies in $N$. Hence $|R| \mid n-1$. Then $|R|=2$ and hence $R=N$, since $n-1 \equiv 2 \bmod 4$, since $n=2 v-1$ and $v=q+1$. Thus $q \equiv 3 \bmod 4$ and we have the assertion (2).

Finally, assume that $n=2 v$. Then $v$ is even by [33, Theorem 13.18]. Thus $q$ must be odd. Assume that $q \equiv 1 \bmod 4$. Let $R$ be defined as above. In this case $8 \mid 2(q+1)$, since $R \cong Q_{2^{m}}, m \geq 3$, must be semiregular on $[B]-\{l\}, n=2(q+1)$ and the unique involution in $G$ is an $(X, l)$-elation, where $X$ is the unique point on $l-\mathscr{O}$ fixed by $G$. This gives a contradiction, hence $q \equiv 3 \bmod 4$. This proves the assertion (3).

It should be stressed that if there exist planes of type (2) with $n$ a prime power then $n$ is actually a prime by Lemma 4.1(1). Furthermore, if there exist planes of type (3) with $n$ a prime power then $n$ is a power of 2 and $q$ is a Mersenne prime by Lemma 4.1(2). Nevertheless, as we shall see in Section 7, in these cases $\Pi$ cannot be the projective extension of a translation plane. 
5.1.2. The case $d_{0}(\bar{G})<v \quad$ In the following we assume that $\bar{G} \not \nexists P S U(3,5)$. Then either $N=N(Q, l), Q \in \Pi-l$, or $N=N(l, l)$ by Lemma 5.4. We treat these two cases separately. In particular, for each of them, we show that $G$ is a perfect central extension of $\bar{G}$. Now, since the groups satisfying $d_{0}(\bar{G})<v$ are listed in Table 1 of Lemma 4.3, we complete this subsection with a case by case investigation.

LEMMA 5.8. If $N=N(Q, l), Q \in \Pi-l$, then $n=23$ and $G \cong S L(2,11)$.

Proof. Assume that $N=N(Q, l)$, where $Q$ is a point of $\Pi-l$ fixed by $G$. Assume also that $G$ fixes a point $P$ of $l-\mathscr{O}$ and let $\Omega$ be the set of $N$-orbits on $Q P-\{Q, P\}$. Then $|\Omega|=(n-1) /|N|$, since $N$ is semiregular on $Q P-\{Q, P\}$. Hence

$$
n=1+|N| \sum_{j \geq 0} \lambda_{j} d_{0}(\bar{G})
$$

where $\lambda_{j} \geq 0, j \geq 0$, and $\sum_{j \geq 0} \lambda_{j}>0$, by Lemma 5.2 .

Assume that $N \not Z Z(G)$. Then $|N| \geq 1+d_{0}(\bar{G})$ by Lemma 5.1. By composing the previous inequality with (5.3) and bearing in mind that $n \leq 2 v$, we obtain $1+d_{0}(\bar{G})+d_{0}(\bar{G})^{2} \leq 2 v$. Now, filtering the groups of Table 1 with respect to the previous inequality, it is easily seen that no cases arise.

It remains to investigate the case where $G$ is a covering group for $\bar{G}$ by Lemma 5.1. The groups $\bar{G} \cong M_{11}$ or $\bar{G} \cong S p(2 h, 2), h>3$, are ruled out by [43, Theorem 5.1.4], since $N \neq\langle 1\rangle$ by our assumption. For the remaining groups, we have $\lambda_{0} \leq 2$ and $\lambda_{j}=0$ for $j>0$ by Lemma 4.3 , since $N \neq\langle 1\rangle$. In particular, $|N| \leq 3$ again by Lemma 4.3(2). Thus either $N \cong Z_{2}$ or $N \cong Z_{3}$ for the groups of the Table 1 by [43, Theorem 5.1.4]. Hence $n=1+|N| \lambda_{0} d_{0}(\bar{G}), \lambda_{0} \in\{1,2\}$ and $|N| \in\{2,3\}$. By Lemma 4.3 and since $v<n \leq 2 v$, it is easily seen that the admissible cases of Table 1 are $G / Z_{2} \cong P S L(2,5)$ and $n=11, G / Z_{2} \cong P S L(2,7)$ and $n=15$, $G / Z_{2} \cong S L(2,9)$ and $n=13, G / Z_{3} \cong P S L(2,9)$ and $n=19, G / Z_{2} \cong P S L(2,11)$ and $n=23, G / Z_{2} \cong P S L(4,2)$ and $n=17, G / Z_{3} \cong A_{7}$ and $n=21, G / Z_{2} \cong A_{7}$ and $n=29, G / Z_{2} \cong H S$ and $n=201$, and $G / Z_{2} \cong S p(6,2)$ and $n=57$.

Actually the cases $G / Z_{3} \cong A_{7}$ and $n=21, G / Z_{2} \cong S p(6,2)$ and $n=57$, and $G \cong H S / Z_{2}$ and $n=201$ cannot occur by [33, Theorem 3.6]. The case $G / Z_{2} \cong P S L(2,7)$ and $n=15$ cannot occur by [31]. If $G / Z_{2} \cong P S L(2,5)$ and $n=11$ then $\Pi \cong P G(2,11)$ and hence $\bar{G} \leq P S L(2,11)$ by [47]. Nevertheless, this case cannot occur, since $\bar{G} \cong P S L(2,5)$ contains involutions fixing a point on $\mathscr{O}$ and hence on $l$, while $P S L(2,11)$ does not.

Assume that $G / Z_{2} \cong S L(2,9)$ and $n=13$. Then $\bar{G} \leq P G L(2,13)$ by [48]. This gives a contradiction, hence $G / Z_{3} \cong P S L(2,9)$ and $n=19$. In this case there exists an involution $\delta$ in $G$ fixing at least 4 points on $l$, since $n+1=20$ and $|\mathscr{O}|=10$. Clearly $\delta \notin N$. Therefore $\delta$ is a Baer collineation of $\Pi$, which is a contradiction. 
Assume that $G / Z_{2} \cong P S L(2,11)$ and $n=23$. Then $G \cong S L(2,11)$ by $[41$, Theorem 7.1.1.(i)]. Since $8 \nmid n-1$, there exists an involution fixing a point on $P Q-\{P, Q\}$. Such an involution must lie outside $N$, which is a contradiction. Assume that $G / Z_{2} \cong P S L(4,2)$ and $n=17$, or $\bar{G} \cong A_{7}$ and $n=29$. Let $J$ be a Sylow 2-subgroup of $G$. Then $2^{6}|| J \mid$ for $G / Z_{2} \cong P S L(4,2)$ and $|J|=2^{4}$ for $G / Z_{2} \cong A_{7}$. Furthermore, $J$ fixes a point $X$ of $\mathscr{O}$, since $|\mathscr{O}|=15$. Then $J_{Z} \neq\langle 1\rangle$ for some $Z \in X Q-\{X, Q\}$, since $|J| \nmid n-1$. In particular, $J_{Z} \cap N=\langle 1\rangle$ and [ $\left.J: J_{Z}\right] \leq 2^{3}$. Hence $J_{Z}$ acts faithfully on $\mathscr{O}$. In particular, $J_{Z}$ contains involutions fixing at least 3 points on $\mathscr{O}$ by [50]. Such involutions are Baer collineations of $\Pi$, since $J_{Z} \cap N=\langle 1\rangle$. Hence $n$ must be a square. This gives a contradiction and therefore $G$ cannot fix points on $l-\mathscr{O}$. Then $l-\mathscr{O}$ consists of nontrivial $\bar{G}$-orbits, since $G$ acts on $l-\mathscr{O}$ as $\bar{G}$. Since each $\bar{G}$-orbit is a multiple of some $d_{i}(\bar{G}), i \geq 0$, we have that $n+1=v+\sum_{j \geq 0} \mu_{j} d_{j}(\bar{G})$, where $\mu_{j} \geq 0, j \geq 0$, and $\sum_{j \geq 0} \mu_{j}>0$. Hence either $n=v+d_{0}(\bar{G})-1$ or $n=2 v-1$ or $n=28$ and $\bar{G} \cong A_{7}$ or $n=30$ and $\bar{G} \cong P S L(4,2)$ by Lemma 4.3 , since $n \leq 2 v$. The latter is ruled out by [33, Theorem 13.18].

Assume that $n=28$ and $\bar{G} \cong A_{7}$. Then $N$ has odd order as $|N| \mid n-1$, so there exists an involution fixing 3 points on $\mathscr{O}$ by [50] and not lying in $N$. Such an involution must be a Baer collineation of $\Pi$ and hence $n$ must be a square. This gives a contradiction.

Assume that $n=v+d_{0}(\bar{G})-1$. Then all the groups of Table 1 , except $\bar{G} \cong$ $P S L(2,9)$ and $n=15, \vec{G} \cong A_{7}$ and $n=21, \bar{G} \cong S p(2 h, 2)$ and $n=2^{2 h}-1$, $G / Z_{2} \cong H S$ and $n=275$, are ruled out by [33, Theorem 13.18]. Nevertheless, the groups $\bar{G} \cong P S L(2,9), \bar{G} \cong A_{7}$ and $\bar{G} \cong S p(2 h, 2)$ cannot occur by [31], by [33, Theorem 3.6], and by [26], respectively. Hence $G / Z_{2} \cong H S$ and $n=275$. Let $X$ be a point on $\mathscr{O}$. Then $\bar{G}_{X} \cong P S U(3,5) \cdot Z_{2}$ by [17, Appendix B]. Now, let $S$ be a Sylow 2-subgroup of $G_{X}$. Clearly $|S|=2^{6}$, since $N \cong Z_{2}$. Then $S$ fixes a point $B$ on $\mathscr{O}-\{X\}$, since $|\mathscr{O}|=176$. Furthermore there exists a non trivial subgroup of $S_{0}$ of $S$ such that $\left[S: S_{0}\right] \leq 2^{2}$, which fixes 3 points on $\mathscr{O}$ and a point on $\Pi-(l \cup\{Q\})$. Thus $S_{0} \cap N=\langle 1\rangle$. Therefore $S_{0}$ contains a Baer collineation of $\Pi$ and hence $n$ must be a square, which is a contradiction.

Assume that $n=2 v-1$. The above arguments rule out the cases $\bar{G} \cong P S L(2,5)$ and $n=11, \bar{G} \cong P S L(2,7)$ and $n=15, \bar{G} \cong S p(2 h, 2)$ and $n=2^{h}\left(2^{h}-1\right)-1$ and $\bar{G} \cong H S$ and $n=351$. Furthermore, the same argument as in Theorem 5.7 rules out the case $\bar{G} \cong P S L(2,9)$ and $n=19$, and the above argument used to rule out $\bar{G} \cong A_{7}$ and $n=29$ may be applied to rule out also the case $\bar{G} \cong P S L(4,2)$ and $n=29$. Finally, the case $\bar{G} \cong M_{11}$ and $n=23$ cannot occur by [43, Theorem 5.1.4], since $N \leq Z_{2} \times Z_{11}$ in this case, which is a contradiction. Thus $G \cong S L(2,11)$ and $n=23$. 
LEMMA 5.9. If $N=N(l, l)$ then either $n=16$ and $G \cong S L(2,7)$ or $n=24$ and $G \cong S L(2,11)$.

Proof. Assume that $\bar{G}$ does not fix any point on $l-\mathscr{O}$. Then $l-\mathscr{O}$ consists of nontrivial $\bar{G}$-orbits. At this point we may use the same argument as in Lemma 5.8 to show that either $n=2 v-1$ or $n=v+d_{0}(\bar{G})-1$ or $n=28$ and $\bar{G} \cong A_{7}$. Actually, the case $n=2 v-1$ is ruled out by the same argument as in Proposition 5.6 part (A). Hence, either $n=v+d_{0}(\bar{G})-1$ or $n=28$ and $\bar{G} \cong A_{7}$.

Assume that $n=28$ and $\bar{G} \cong A_{7}$. If $|N| \geq 4$, we may apply the same argument as in Lemma 5.4 to rule out this case. Hence $N \cong Z_{2}$. Then $|J|=2^{4}$, where $J$ is any Sylow 2-subgroup of $G$. Furthermore, $J$ fixes a point $O$ of $\mathscr{O}$, since $|\mathscr{O}|=15$. Then $J_{m} \neq\langle 1\rangle$ for some $m \in[O]-\{l\}$, since $|J| \nmid n$. In particular, $J_{m} \cap N=\langle 1\rangle$ and $\left[J: J_{m}\right] \leq 2^{3}$. Hence $J_{m}$ acts faithfully on $\mathscr{O}$. In particular, $J_{m}$ contains involutions fixing at least 3 points on $\mathscr{O}$ by [50]. Such involutions are Baer collineations of $\Pi$, since $J_{m} \cap N=\langle 1\rangle$. Hence $n$ must be a square. This gives a contradiction.

Assume that $n=v+d_{0}(\bar{G})-1$. Then each case of Table 1 , except $\bar{G} \cong H S$ and $n=275$, is ruled out by the same argument as in Lemma 5.8. In the remaining case, $N$ has odd order as $|N| \mid n^{2}$ and $n$ is odd. Hence, it is easily seen that there exists an involution in $G$ which is Baer collineation of $\Pi$. This gives a contradiction, since $n=275$, so we may assume that $G$ fixes at least a point $X$ on $l-\mathscr{O}$. Assume that $|N|>n$. Then $N(X, l)<N$. Let $\Psi$ be the set of $N$-orbits on $[X]-\{l\}$. Note that each $N$-orbit on $[X]-\{l\}$ has length $[N: N(X, l)]$, since $N=N(l, l)$ and $N(X, l)<N$. Clearly $G$ acts on $\Psi$ as $N \triangleleft G$. Then $|\Psi| \geq d_{0}(\bar{G})$ by Lemma 5.2. This gives a contradiction, since $|\Psi| \leq 3$ by Lemma 4.5 , as $|N|>n$. Thus $|N| \mid n$.

Assume that $N \not Z Z(G)$. There exists a Sylow $t$-subgroup $S$ of $N$ such that $\bar{G} \leq S L(V)$, where $V=S / \Phi(S)$ by Lemma 5.1. We have $|V| \geq b$, where $b=t^{r(\bar{G})}$ and $r(\bar{G})$ is a suitable lower bound for $\operatorname{dim}_{G F(t)}(V)$. Indeed, such a lower bound can be easily recovered from [43], in particular it can be recovered from Theorem 5.3.9 and Proposition 5.4.13 when $\bar{G}$ is classical, from Proposition 5.3.7 when $\bar{G} \cong A_{7}$, and from Proposition 5.3.8 when $\bar{G} \cong H S$ or $\bar{G} \cong M_{11}$. This information must be combined in some cases with [11] in order to determine $b$ as follows: pick $\bar{G} \cong M_{11}$ for example, then $r\left(M_{11}\right)=5$ by [43, Proposition 5.3.8]. Hence $|V| \geq t^{5}$. From [11], we see that $t>2$. Hence $b=3^{5}$ and $|V| \geq 3^{5}$. The same argument can be repeated for each group listed in the Table 1 . Then $|V| \geq b$ and hence $|N| \geq b$ as $V=S / \Phi(S)$ and $S \leq N$. On the other hand, we must have $b \leq n$, since $|N| \mid n$. By a direct inspection of the Table 1 , we see that $\bar{G} \cong P S L(2,7)$ and $|N| \in\{8,16\}$, $\bar{G} \cong P S L(4,2)$ or $\bar{G} \cong A_{7}$ and $|N|=16, \bar{G} \cong S p(2 h, 2), h \geq 3$, and $2^{2 h}|| N \mid$ are the unique cases satisfying the inequality $b \leq n$.

Assume that $\bar{G} \cong P S L(2,7)$. Then $n=16$, since $9 \leq n \leq 16,|N| \mid n$ and $|N| \in\{8,16\}$. Let $C \leq G$ be such that $C \cong Z_{7}$. Then $\operatorname{Fix}(C)$ fixes a subplane 
of $\Pi$ of order at least 2 , since $n \equiv 2 \bmod 7$. Actually, $\operatorname{Fix}(C) \cong P G(2,2)$ by $[33$, Theorem 3.7], since $n=16$. Thus $\bar{G}$ cannot fix $l-\mathscr{O}$ pointwise. Hence $l-\mathscr{O}$ consists of either a $\bar{G}$-orbit of length 7 plus two points fixed by $\bar{G}$, or a $\bar{G}$-orbit of length 8 plus one point fixed by $\bar{G}$ by $\left[17\right.$, Appendix B], since $\bar{G} \cong P S L(2,7)$. Let $T \leq N_{G}(C)$ be such that $T \cong Z_{3}$. Then Fix $(C) \subset \operatorname{Fix}(T)$, since $T$ fixes two points $l \cap \operatorname{Fix}(C)$ at least in any of the two possible orbital configurations of $l-\mathscr{O}$. This implies that $T$ must fix a further point on $l$, since $|l-(l \cap \operatorname{Fix}(C))|=14$. So $\operatorname{Fix}(T) \cong P G(2,4)$ by [33, Theorem 3.7], since $n=16$. Since a $Z_{3}$ normalizes exactly one $Z_{7}$ in $\bar{G}$, there exists a point $A \in \operatorname{Fix}(T)-(\operatorname{Fix}(C) \cup l)$ such that $Z_{3} \leq G_{A}\left\lfloor Z_{7} . Z_{3}\right.$. Then $Z_{3} \leq G_{A^{N}} \not \leq Z_{7} . Z_{3}$. Let $\Omega$ be the set of $N$ orbits on $\Pi-l$. Then $16^{2} /|N|=\lambda_{1} 7+\lambda_{2} 8$, with $\lambda_{1}, \lambda_{2} \geq 0$ and $\lambda_{1}+\lambda_{2}>0$, by Lemma 5.2, since $|\Omega|=16^{2} /|N|$. Note that $\lambda_{1}>0$ since $Z_{3} \leq G_{A^{N}} \nsubseteq Z_{7} . Z_{3}$. Also $\lambda_{2}>0$, since $G_{B^{N}} \cong Z_{7} . Z_{3}$ for any $B \in \operatorname{Fix}(C)-l$. It is a straightforward calculation to show that the above Diophantine equation has solutions only for $|N| \leq 4$ which is a contradiction.

Assume that $\bar{G} \cong P S L(4,2)$ or $\bar{G} \cong A_{7}$ and $|N|=16$. Then $n=16$ as $|N| \mid n$ and $15<n \leq 30$. Hence there exists $H<G_{O}$, where $O \in \mathscr{O}$, such that $H \cong P S L(2,7)$ and $H \cap N=\langle 1\rangle$, since $N$ is regular on [O] $-\{l\}$ as $|N|=16$. Clearly $\Pi$ cannot be Desarguesian, since the full collineation group induced on a line is $P \Gamma L(2,16)$ and $A_{7} \nless P \Gamma L(2,16)$. Then $\Pi$ is either the Lorimer-Rahilly plane or the Johnson-Walker plane or their duals by [15]. This gives a contradiction, since the full collineation group induced on a line in any of these planes is isomorphic to $\operatorname{PSL}(2,7) \times S_{3}$ by [37].

Assume that $\bar{G} \cong S p(2 h, 2), h \geq 3$, and $2^{2 h}|| N \mid$. Then $n=2^{2 h}$, since $|N| \mid n$ and $v<n \leq 2 v$ with $v=2^{h-1}\left(2^{h}+1\right)$. Then $G_{O}=G_{O, e} N$ and $G_{O, e} \cap N=\langle 1\rangle$ for some line $e \in[O]-\{l\}$, since $N$ is regular on $[O]-\{l\}$ as $N=N(X, l)$ with $X \in l-\mathscr{O}$. Then there exists an involution in $G_{O, e}$ fixing $2^{2 h-2}$ points on $\mathscr{O}$ by [17, Example 5.4.3]. This gives a contradiction by [33, Theorem 3.7]. Hence $G$ is a covering group for $G$ by Lemma 5.1.

Note that the groups $\bar{G} \cong M_{11}$ and $\bar{G} \cong S p(2 h, 2), h>3$, are ruled out by [43, Theorem 5.1.4], since $N \neq\langle 1\rangle$ by our assumption. In particular, $N$ is cyclic and $|N| \leq 3$ for the remaining groups of Table 1 by [43, Theorem 5.1.4]. As a consequence, $N=N(X, l)$. Assume that $G$ fixes a further point $Y$ on $l-\mathscr{O}$. Then $G$ acts on the set $\Psi$ of $N$-orbits on $[Y]-\{l\}$. Then $|\Psi|=n /|N|$ as $N(Y, l)=\langle 1\rangle$. Then $n=|N| \sum_{j \geq 0} \theta_{j} d_{j}(\vec{G})$, with $\theta_{j} \geq 0, j \geq 0$, and $\sum_{j \geq 0} \theta_{j}>0$ by Lemma 5.2. Actually, $\theta_{j}=0$ for $j>1$ and $\left(|N|, \theta_{0}, \theta_{1}\right)=(2,0,1)$ or $(2,1,0)$, or $\left(|N|, \theta_{0}, \theta_{1}\right)=(3,1,0)$ and $\bar{G} \cong P S L(2,9)$, or $\bar{G} \cong A_{7}$, or $\left(|N|, \theta_{0}, \theta_{1}\right)=(2,2,0)$ and $\bar{G} \cong A_{7}$ by Lemma 4.3, since $N \neq\langle 1\rangle$ and $v<n \leq 2 v$. Let $r \in[Y]-\{l\}$. Then $G_{r^{N}}=G_{r} N$ and $G_{r} \cap N=\langle 1\rangle$. In particular, $G_{r} \cong \bar{G}_{r^{N}}$, where $\bar{G}_{r^{N}}$ is the stabilizer in a $\bar{G}$-orbit on $\Psi$ of length $d_{0}(\bar{G})$ or $2 d_{0}(\bar{G})$ or $v$.

If $\left|G_{r}\right|$ is even then $G_{r}$ contains involutions which are Baer collineations of $\Pi$, since they fix the points $X$ and $Y$ on $l$ and $n$ is even. So $n=|N|\left(\theta_{0} d_{0}(\bar{G})+\theta_{1} v\right)$ 
must be a square. It is a plain to see that the only groups in Table 1 satisfying one of the previous numerical conditions are $G \cong S L(2,7)$ or $G / Z_{2} \cong P S L(4,2)$, and $n=16$. Nevertheless, the latter is ruled out by the same argument as above, since $P S L(2,7) \leq G_{r}$. Therefore $G \cong S L(2,7)$ and $n=16$. Let $C$ and $T$ be defined as above. The above argument still works to show that $\operatorname{Fix}(C) \subset \operatorname{Fix}(T)$, with Fix $(C) \cong P G(2,2)$ and $\operatorname{Fix}(T) \cong P G(2,4)$. This, in particular, still forces $l-\mathscr{O}$ to consist of a $\bar{G}$-orbit of length 7 plus 2 points fixed by $G$, again by the above argument. Now, Let $D \leq N_{G}(T)$ be such that $D \cong Z_{4}$ as $G \cong S L(2,7)$. Clearly $D$ acts on Fix $(T)$ and $D$ fixes exactly 3 points on $l \cap$ Fix $(T)$. In particular, $D \leq P \Gamma L(3,4)$ as $\operatorname{Fix}(T) \cong P G(2,4)$. This gives a contradiction, since $P \Gamma L(3,4)$ contains no cyclic subgroups of order 4 fixing exactly three points on a line.

Assume that $\left|G_{r}\right|$ is odd. Then $G \cong S L(2,11)$ and $n=24$ by a direct inspection of Table 1. Let $L \leq G$ be such that $L \cong Z_{11}$. Then $L$ fixes a subplane of $\Pi$ of order at least 2, since $n+1=25$ and since $G$ fixes the points $X$ and $Y$ on $l-\mathscr{O}$. Actually, $o(\operatorname{Fix}(L))=2$ by [33, Theorem 3.7], since $n=24$. Let $T \leq N_{G}(L)$ be such that $T \cong Z_{5}$. Clearly $T$ acts nontrivially on $\operatorname{Fix}(L)$. Hence $T \leq P S L(3,2)$, since $\operatorname{Fix}(L) \cong P G(2,2)$. This gives a contradiction, hence $X$ is the unique point on $l-\mathscr{O}$ which is fixed by $G$. Thus either $n=v+d_{0}(\bar{G})$ or $n=2 v$ or $n=29$ and $\bar{G} \cong A_{7}$. Assume that the latter occurs. Then $G-N$ contains an involution, as $|N| \mid n$ and $n$ is odd. This involution is a Baer collineation of $\Pi$, since it fixes three points on $\mathscr{O}$ by [50]. This gives a contradiction.

Assume that $n=v+d_{0}(\bar{G})$. The cases $\bar{G} \cong A_{7}$ and $n=22$ and $\bar{G} \cong A_{8}$ and $n=23$ are ruled out by [33, Theorem 13.18] and by [26], respectively. Again, we may apply a similar argument to that of Lemma 5.8 in order to rule out the cases $G \cong S L(2,11)$ and $n=23$ or $\bar{G} \cong H S$ and $n=276$ (in this case $n$ is even and there also exists a 2-subgroup of $G_{A}, A \in \mathscr{O}$, of order at least 4, fixing two points on $\mathscr{O}$ and two on $\Pi-l$ ). Hence $G \cong S L(2,9)$ and $n=16$. Let $U \leq G$ be such that $U \cong E_{9}$. Clearly $N_{G}(U) \cong U . T$, where $T \cong Z_{8}$, since $G \cong S L(2,9)$. In particular $N_{G}(U)$ fixes a point on $R$ on $\mathscr{O}$, since $|\mathscr{O}|=10$. Then $U$ fixes at least a line $r$ of $[R]-\{l\}$, since $n=16$ and $U \cong E_{9}$. Note that $T$ acts semiregularly on $[R]-\{l\}$, since the unique involution of $T$ generates $N$ and $N=N(X, l)$ with $X \in l-\mathscr{O}$. Thus $\left|r^{T}\right|=8$. Moreover, $r^{T} \subset \operatorname{Fix}(U)$ since $T \leq N_{G}(U)$ and $r \in \operatorname{Fix}(U)$. Hence $T$ fixes at least one point on $s-\{R\}$ for each $s \in r^{T}$, since $n=16$. In particular there exists a non trivial subgroup $U_{0}$ of $U$ such that $\left[U: U_{0}\right] \leq 3$ fixing at least three points on $l$. Therefore $\operatorname{Fix}\left(U_{0}\right)$ is a subplane of $\Pi$, since $\operatorname{Fix}(U) \subset \operatorname{Fix}\left(U_{0}\right)$. In particular $o\left(\operatorname{Fix}\left(U_{0}\right)\right) \geq 7$, since $r^{T} \cup\{l\} \subset[R] \cap \operatorname{Fix}\left(U_{0}\right)$. This gives a contradiction by [33, Theorem 3.7] since $n=16$.

Assume that $n=2 v$. Note that any admissible case of Table 1 , except $G \cong S L(2,7)$ and $n=16$ or $G \cong S L(2,11)$ and $n=24$, is ruled out by arguments similar to those used above. This proves the assertion. 
Now assume that the case $\bar{G} \cong P S U(3,5)$ is admissible. The following theorem completes this subsection and shows that the assertions (2) and (3) of Theorem 1.1 are true when $d_{0}(\operatorname{soc}(\bar{G}))<v$.

THEOREM 5.10. One of the following occurs:

(1) $n=16$ and $G \cong S L(2,7)$;

(2) $n=23$ or 24 and $G \cong S L(2,11)$.

Proof. It remains to rule out the group $\bar{G} \cong P S U(3,5)$ in order to prove this theorem by Propositions 5.8 and 5.9. Assume that $N \not Z Z(G)$. Then there exists a Sylow $t$-subgroup $S$ of $N$ such that $\bar{G} \leq S L(V)$, where $V=S / \Phi(S)$ by Lemma 5.1. Then either $|V| \geq 2^{20}$ by [43, Theorem 5.3.9] when $5 \nmid|V|$, or $5^{6}|| V \mid$ by [43, Proposition 5.4.13]. Hence either $|N| \geq 2^{20}$ or $5^{6}|| N \mid$. On the other hand, either $|N| \mid n-1$ or $|N| \mid n^{2}$ by Lemma 4.5. By composing all these bounds on the order of $N$, we see that the unique admissible case is $5^{6}|| N \mid$ and $|N| \mid n^{2}$, since $n=2 v$ and $v=126$. This yields $n=250$, since $5^{3} \mid n$ and $126<n \leq 252$. This gives a contradiction by [33, Theorem 13.18]. Hence $G$ is a covering group for $P S U(3,5)$. Thus $N \cong Z_{3}$ by $[43$, Theorem 5.1.4], since $N \neq\langle 1\rangle$ by our assumption. Thus any involution $\phi$ in $G$ actually lies in $G-N$, so, it is well known that $\phi$ fixes exactly six points on $\mathscr{O}$. Hence $n$ is a square. Therefore $\sqrt{n} \in\{12,13,15\}$, since $126<n \leq 252$ and since $\sqrt{n}=14$ cannot occur by [33, Theorem 3.6]. Note that $C_{G}(\phi)$ is non solvable, since it has a section which is isomorphic to $\operatorname{PGL}(2,5)$. Thus the cases $\sqrt{n}=12$ or 15 are ruled out by [36] and [31], respectively. Hence $\sqrt{n}=13$. Then $\operatorname{Fix}(\phi) \cong P G(2,13)$ by [48]. Denote by $\bar{C}_{G}(\phi)$ the group induced on Fix $(\phi)$ by $C_{G}(\phi)$. Then $\bar{C}_{G}(\phi)$ acts trivially on Fix $(\phi)$, since 5|| $\bar{C}_{G}(\phi) \mid$ while $5 \nmid|P G L(3,13)|$. Thus there exists an element of order 5 in $P S U(3,5)$ fixing the same 6 points on $\mathscr{O}$ fixed by $\phi$. This gives a contradiction.

5.2. The faithful case In this subsection we deal with case $N=\langle 1\rangle$. Since $G$ is simple, either $K=\langle 1\rangle$ and hence $G$ has a non trivial orbit on $l-\mathscr{O}$, or $G=K$ and hence $G$ fixes $l-\mathscr{O}$ pointwise.

This subsection is structured as follows. If $G$ has a non trivial orbit on $l-\mathscr{O}$, we reduce to the case $d_{0}(G)<v$ by using the arguments of parts (C) and (D) of Proposition's 5.5 and 5.6, respectively. Then we show that the involutions in $G$ are Baer collineations of $\Pi$ by using the results of Ho and Gonçalves [32]. Finally, a case by case investigation shows that $\Pi$ is the Johnson-Walker translation plane of order 16 or its dual, and $G \cong P S L(2,7)$. If $G$ fixes $l-\mathscr{O}$ pointwise, we reduce to the case $n=v+1$ by using Lemma 4.2. At this point we show that $G$ admits another 2-transitive orbit of length $v$ not contained in a line which is in contrast with the order $n$ of $\Pi$. 
PROPOSITION 5.11. If there exists a non trivial $G$-orbit on $l-\mathscr{O}$ then $\Pi$ is the Johnson-Walker translation plane of order 16 or its dual, and $G \cong P S L(2,7)$.

PROOF. Assume there exists a non trivial $G$-orbit $\mathscr{O}^{\prime}$ on $l-\mathscr{O}$, so $n \geq v+d_{0}(G)$. If $d_{0}(G)=v$ then either $n=2 v-1$ or $n=2 v$. At this point we may use the arguments of parts (C) and (D) of Propositions 5.5 and 5.6, respectively, to rule out these cases. Hence $d_{0}(G)<v$.

Assume that $G$ contains an involutory perspectivity. If there exists a point $P \in \Pi-l$ such that $G_{P}=\langle 1\rangle$ then $|G| \leq|\Pi-l|$. This yields $v(v-1) \theta \leq 4 v^{2}$, since $n \leq 2 v$ and since $|G|=v(v-1) \theta, \theta \geq 1$. Thus either $\theta=5$ and $v=5$, or $\theta \leq 4$. Then either $G \cong P S L(2,5)$ and $n=11$ or $n=12$, or $G \cong P S L(2,7)$ and $n=15$ or $n=16$ by direct inspection of Table 1 . Nevertheless the former cannot occur, respectively, by [47], since $|\mathscr{O}|=6$, and by [36]. Also the latter cannot occur, respectively, by [31], and by [15], since $G$ contains involutory perspectivities and $|\mathscr{O}|=8$. Hence $G$ is totally irregular on $\Pi$. Then $G \cong P S L(2,5)$ and $n=11$ or $n=12$, or $G \cong P S L(2,7)$ and $n=15$ or $n=16$ by [32, Theorems 1 and 2]. Again this gives a contradiction.

Assume that each involution is a Baer collineation of $\Pi$. So $n$ must be a square. Then either $G \cong P S L(2,7)$ or $G \cong P S L(2,9)$ and $n=16$, or $G \cong A_{7}$ or $G \cong A_{8}$ and $n=25$, or $G \cong P S U(3,5)$ and $n \in\left\{13^{2}, 14^{2}, 15^{2}\right\}$, or $G \cong H S$ and $n \in\left\{17^{2}, 18^{2}\right\}$, or $G \cong S p(2 h, 2), h \geq 3$ and $n=2^{2 h}$. Actually, the cases $G \cong P S U(3,5)$ and $n \in\left\{13^{2}, 14^{2}, 15^{2}\right\}$ cannot occur by the same argument of Theorem 5.10. The case $G \cong S p(2 h, 2), h \geq 3$, and $n=2^{2 h}$ cannot occur by [33, Theorem 3.7], since $G$ contains Baer involutions fixing $2^{2 h-2}$ points on $\mathscr{O}$ by [17, Example 5.4.3].

Assume that $G \cong A_{7}$ and $n=25$. Then there exists an involution in $G$ fixing at least seven points on $l$ by [50] and since $\left|l-\left(\mathscr{O} \cup \mathscr{O}^{\prime}\right)\right|=4$. This gives a contradiction by [33, Theorem 3.7]. Assume that $G \cong A_{8}$ and $n=25$. Then there exists an involution in $G$ fixing at least 10 points on $l$ by [50] and since $\left|l-\left(\mathscr{O} \cup \mathscr{O}^{\prime}\right)\right|=3$. This gives a contradiction by [33, Theorem 3.7].

Assume that $G \cong \operatorname{PSL}(2,9)$ and $n=16$. Set $\{X\}=l-\left(\mathscr{O} \cup \mathscr{O}^{\prime}\right)$. Let $S$ be a Sylow 2-subgroup of $G$. Then $S=\langle\alpha, \beta\rangle$ with $\alpha^{4}=1, \beta^{2}=1$ and $\alpha^{\beta}=\alpha^{-1}$. Note that $|\operatorname{Fix}(\alpha) \cap l|=3,\left|\operatorname{Fix}\left(\alpha^{2}\right) \cap l\right|=5$ and $|\operatorname{Fix}(\beta) \cap l|=5$, since $l=\mathscr{O} \cup \mathscr{O}^{\prime} \cup\{X\}$, and since $G \cong P S L(2,9)$ acts in its 2-transitive permutation representations of degree 10 and 6 on $\mathscr{O}$ and on $\mathscr{O}^{\prime}$, respectively. Furthermore, $\left|\operatorname{Fix}\left(\alpha^{2}\right) \cap \operatorname{Fix}(\beta) \cap l\right|=3$. This yields $\operatorname{Fix}\left(\alpha^{2}\right) \cong \operatorname{Fix}(\beta) \cong P G(2,4)$ and $\operatorname{Fix}(\alpha) \cong P G(2,2)$ with $\operatorname{Fix}(\alpha) \subset \operatorname{Fix}\left(\alpha^{2}\right)$. Moreover, $\operatorname{Fix}\left(\alpha^{2}\right) \cap \operatorname{Fix}(\beta) \cong P G(2,2)$ and $\operatorname{Fix}(\alpha) \cap \operatorname{Fix}(\beta)$ consists of three collinear points of $\operatorname{Fix}\left(\alpha^{2}\right)$ including $X$. Thus $\left|\operatorname{Fix}\left(\alpha^{2}\right)-(\operatorname{Fix}(\alpha) \cup \operatorname{Fix}(\beta) \cup l)\right|=10$. Let $U \leq G$ be such that $U \cong E_{9}$. It is easily seen that $\operatorname{Fix}(U)$ fixes exactly two points on $l$, since the permutation $\gamma=(123)(456)$ lies in $U$ and $\gamma$ is fixed point free on $\mathscr{O}^{\prime}$. Thus $\operatorname{Fix}(U)$ cannot be a subplane of $\Pi$. Then there exists a line $r$ of $\Pi$ such that $\operatorname{Fix}(U)-l \subset r$. In particular $\operatorname{Fix}(G) \subset \operatorname{Fix}(U)$ and $|\operatorname{Fix}(U) \cap \operatorname{Fix}(\alpha)-l| \leq 3$. 
Hence, there are at least two points of $\Pi-l$ (lying in Fix $(\alpha)-l$ ), say $X_{1}$ and $X_{2}$, such that $G_{X_{1}} \cong Z_{2}$ and $G_{X_{2}} \cong Z_{4}$, since $\operatorname{Fix}(G) \subset \operatorname{Fix}(U)$ and $\operatorname{Fix}(U)-l \subset r$ and Fix $(\alpha) \cap \operatorname{Fix}(\beta)$ consists of 3 collinear points of Fix $\left(\alpha^{2}\right)$ including $X$ and there are no proper subgroups of $G$ of order divisible by 20 . Then $|\Pi-l| \geq 270$, since $X_{1}^{G} \cup X_{2}^{G} \subset \Pi-l$ with $\left|X_{1}^{G}\right|=180$ and $\left|X_{2}^{G}\right|=90$. This gives a contradiction, since $n=16$.

Finally, if $G \cong P S L(2,7)$ and $n=16$ then $\Pi$ is the Johnson-Walker translation plane of order 16 or its dual and the $G$-orbits on $l$ have lengths $8,7,1$ and 1 by [15]. This proves the assertion.

THEOREM 5.12. Let $\Pi$ be a projective plane of order $n$ and let $\mathscr{O}$ be a 2-transitive $G$-orbit of length $v$ on a line with $n>v \geq n / 2$. If $G$ is almost simple and $G$ is faithful on $\mathscr{O}$ then $\Pi$ is the Johnson-Walker translation plane of order 16 or its dual and $G \cong P S L(2,7)$.

Proof. Assume that $G$ fixes $l-\mathscr{O}$ pointwise. Assume also that $n \geq v+2$. Thus any involution in $G$ is a Baer collineation of $\Pi$, since $|l-\mathscr{O}| \geq 3$. Then $n+1-v \leq \sqrt{n}+1$ and hence $v+2 \leq n \leq v+\sqrt{2 v}$, as $n \leq 2 v$. Suppose there exists a point $Y$ on $l-\mathscr{O}$ such that $G$ admits an orbit $\mathscr{O}^{*}$ of length $v$ on $[Y]-\{l\}$. If $G$ also admits a further non trivial $G$-orbit on $[Y]-\{l]$ then $\Pi$ has order 16 and $G \cong P S L(2,7)$ and $v=8$ by the dual of Proposition 5.11. This gives a contradiction by [15], since $G$ fixes $l-\mathscr{O}$ pointwise and $|l-\mathscr{O}|=9$ in this case. Then $G$ fixes $[Y]-\left(\{l\} \cup \mathscr{O}^{*}\right)$ linewise. If there exists a line $r$ in $[Y]-\left(\{l\} \cup \mathscr{O}^{*}\right)$ such that $G$ fixes two points on $r-\{Y\}$ then $G$ is planar on $\Pi$. In particular $o(\operatorname{Fix}(G))=|l-\mathscr{O}|-1$ since $G$ is transitive on $\mathscr{O}$ and $G$ fixes $l-\mathscr{O}$ pointwise. Then $G_{o}$ is planar on $\Pi$ since $\operatorname{Fix}(G) \subset \operatorname{Fix}\left(G_{o}\right)$. Furthermore, $o\left(\operatorname{Fix}\left(G_{o}\right)\right)=|l-\mathscr{O}|$ since $G$ is 2-transitive on $\mathscr{O}$. So $o\left(\operatorname{Fix}\left(G_{o}\right)=o(\operatorname{Fix}(G))+1\right.$. This gives a contradiction by [33, Theorem 3.7]. Note that $G$ and $G_{O}$ are still planar if $G$ fixes a point, other than $Y$, on at least two distinct lines of $[Y]-\left(\{l\} \cup \mathscr{O}^{*}\right)$, so this case also cannot occur. Hence there exists at least a line $m$ in $[Y]-\left(\{l\} \cup \mathscr{O}^{*}\right)$ on which $G$ does not fix any point, since $\left|[Y]-\left(\{l\} \cup \mathscr{O}^{*}\right)\right| \geq 2$ as $n \geq v+2$. Then $m-\{Y\}$ consists of nontrivial $G$-orbits. It should be stressed that $G$ cannot admit orbits of length $v$ on $m-\{Y\}$, otherwise $\Pi$ has order 16 and $G \cong P S L(2,7)$ and $v=8$ by Proposition 5.11, in contrast with the above argument. Let $P \in m-\{Y\}$. Then $\left|P^{G}\right|>1$, since $m-\{Y\}$ consists of nontrivial $G$-orbits. Clearly $\left|P^{G}\right|=\lambda d_{k}(G)$ for some primitive permutation representation degree $d_{k}(G), k \geq 0$, of $G$.

Assume that $d_{0}(G)=v$. Clearly $G \not \equiv S p(2 h, 2), h \geq 3$, by the same argument as in Proposition 5.11. Then $\lambda v \leq\left|P^{G}\right| \leq v+\sqrt{2 v}$, since $P^{G} \subset m-\{Y\}$ and $n \leq v+\sqrt{2 v}$ and $d_{k}(G)>v$. Thus $\lambda=1$ and $\left|P^{G}\right|=v+1$ by Lemma 4.2. Since $n \geq v+2$, there exists $Q \in m-\left(\{Y\} \cup P^{G}\right)$. Then $\left|Q^{G}\right|>1$, since $m-\{Y\}$ consists of nontrivial $G$-orbits. Then $\left|Q^{G}\right|=v+1$ by the previous argument with $Q$ in the 
role of $P$. So $n \geq 2 v+2$, since $P^{G} \cup Q^{G} \subset m-\{Y\}$. This gives a contradiction.

Assume that $d_{0}(G)<v$. Note that the above argument yields $\left|P^{G}\right|=\lambda d_{0}(G)$ by Lemma 4.3 and since $G$ cannot admit orbits of length $v$ on $m-\{Y\}$. Therefore every admissible nontrivial $G$-orbit on $m-\{Y\}$ must be a multiple of $d_{0}(G)$. This implies $n=\theta d_{0}(G), \theta \geq 1$. Moreover, $n$ must be a square and $v+2 \leq n \leq v+\sqrt{2 v}$. Now by a direct inspection of the Table 1 , it is easily seen that no cases arise. Thus $[Y]-\{l\}$ cannot contain an orbit of length $v$ for any $Y \in l-\mathscr{O}$. At this point we may use the previous argument to show that for any $Z \in l-\mathscr{O}$, the set $[Z]-\{l\}$ consists of a $G$-orbit of length $v+1$ plus a line fixed by $G$, since $G$ cannot be planar on $\Pi$. In particular $G$ is one of the exception groups listed in Lemma 4.2. Then $n=9$ and $G \cong P S L(2,7)$, since $n$ must be a square. This gives a contradiction by [30, Theorem A], since $G$ contains Baer collineations of $\Pi$.

Assume that $n<v+2$. That is $n=v+1$, since $n>v$. Note that $G$ fixes exactly a triangle $\Delta$ having $l$ as its side. In particular each side of $\Delta$ consists of the vertices of $\Delta$ which are fixed by $G$ and of a 2-transitive $G$-orbit of length $v$. This implies that $G_{O}$ fixes a subplane of $\Pi$ isomorphic to $P G(2,2)$. Then there exists a point $Q \in \operatorname{Fix}\left(G_{o}\right)-(l \cup \Delta)$ such that $\left|Q^{G}\right|=v$. Clearly $Q^{G}$ is not contained in a line, since $Q^{G} \subset \Pi-(l \cup \Delta)$ and Fix $(G)=\Delta$. If $Q^{G}$ is a $v$-arc then $Q^{G} \cup \Delta$ is a hyperoval. Then $G \cong P S L\left(2,2^{s}\right), s \geq 2$, or $G \cong S z\left(2^{s}\right), s \geq 3, s$ odd, or $G \cong P S L\left(2,2^{s}\right)$, $s \geq 2$, by [7, Main Theorem]. Thus $n=2^{i s}+2, i \in\{1,2,3\}$, respectively, and $s \geq 2$. This yields $n \equiv 2 \bmod 4$. This gives a contradiction by [33, Theorem 13.18]. Hence $Q^{G}$ is the set of points of a nontrivial 2- $(v, k, 1)$ design $\mathscr{D}$ (see the preliminaries of [4]). By [40, Theorem 1], we have that either $\mathscr{D} \cong P G(2, q), G \cong P S L(3, q)$ and hence $n=q^{2}+q+2$, or $\mathscr{D}$ is Hermitian Unital, $G \cong P S U(3, q), q>2$, and hence $n=q^{3}+2$, or $\mathscr{D}$ is Ree Unital and $G \cong{ }^{2} G_{2}(q), q=3^{2 m+1}, m>1$, and hence $n=q^{3}+2$. If $q$ is even, then $n \equiv 2 \bmod 4$ as $q>2$ (clearly the case $G \cong P S L(3,2)$ and $n=8$ cannot occur). This is impossible by [33, Theorem 13.18]. Hence $q$ is odd.

Now, it is easily seen that $G$ contains an involution fixing the 2 points of $l-\mathscr{O}$ and either exactly $q+1$ points on $\mathscr{O}$ when $\mathscr{D}$ is a Unital, or exactly $q+2$ points on $\mathscr{O}$ when $\mathscr{D} \cong P G(2, q)$. So, either $n=(q+2)^{2}$ or $n=(q+3)^{2}$ by [33, Theorem 3.7]. This gives a contradiction in any case. Thus $G$ cannot fix $l-\mathscr{O}$ pointwise and hence the assertion follows by Proposition 5.11.

This completes the proof of parts (2) and (3) of Theorem 1.1.

\section{The affine case}

Throughout this section $\operatorname{soc}(\bar{G})$ is assumed to be an elementary abelian $p$-group for some prime $p$. Hence $\mathscr{O}$ is endowed with the structure of a $G F(p)$-vector space and the zero vector in $\mathscr{O}$ is denoted by $O$. Let $|\mathscr{O}|=p^{d}, p$ prime, $d \geq 1$. Then $\bar{G}=\bar{T} \bar{G}_{O}$, 
where $\bar{T}$ is the whole translation group of $\mathscr{O}$ and $\bar{G}_{O} \leq \Gamma L(t, p)$. By [28], a structure of $d^{*}$-dimensional vector space $V$ over a field $L \cong G F\left(p^{h}\right), h \mid d, d=h d^{*}$, may be defined on $\mathscr{O}$ in such a way that $\bar{G} \leq A \Gamma L\left(d^{*}, p^{h}\right)$ and $O$ is identified with the zero-vector of $V$.

6.1. The faithful case. Assume that $N=\langle 1\rangle$. Then $\bar{G}=G$ and hence $G=T G_{O}$. In this subsection we prove the following result whose proof relies essentially on Theorem 2.1.

THEOREM 6.1. If $v \notin\left\{5^{2}, 7^{2}, 11^{2}, 29^{2}, 59^{2}\right\}$ then $G \leq A \Gamma L(1, v)$ and one of the following occurs:

(1) $n=v+1, v=2^{d}$ or $v \equiv 3 \bmod 4$;

(2) $n=2 v-1$;

(3) $n=2 v, v=2^{d}$;

(4) $n-\sqrt{n}+1=v$ and $v$ is a prime.

PROOF. Let $K$ be the kernel of the representation of $G$ on $l-\mathscr{O}$. Since $G$ is primitive on $\mathscr{O}$, either $K=\langle 1\rangle$ or $T \leq K \leq G$ by [17, Theorem 4.3B], since $T=\operatorname{soc}(G)$. Assume that $K=\langle 1\rangle$. Then there exists $X \in l-\mathscr{O}$ such that the kernel of the action of $G$ on $X^{G}$ is trivial, again by [17, Theorem 4.3B]. Set $\mathscr{O}^{\prime}=X^{G}$. Then $v|| \mathscr{O}^{\prime} \mid$ by the O'Nan-Scott theorem (see, for example, [17, Theorem 4.1A]), since $v=|T|$. Thus $n \geq 2 p^{d}-1$, since $\mathscr{O} \cup \mathscr{O}^{\prime} \subset l$ and $v=p^{d}$. Actually, either $n=2 p^{d}-1$ or $n=2 p^{d}$, since $n \leq 2 p^{d}$. In particular, we deduce that the action of $G$ on $\mathscr{O}$ and on $\mathscr{O}^{\prime}$ is the same. Assume that $G$ contains a Baer involution of $\Pi$. Then $n$ is a square. If $n=2 p^{d}-1$ then either $(n, p, d)=\left(239^{2}, 13,4\right)$ or $p$ is odd and $d \leq 2$ by [54, Results A11.1 and the result of page 141]. Nevertheless, the former is ruled out by [30, Theorem A]. Thus $d \leq 2$ and hence $d^{*} \leq 2$. At this point we may use [28, Lemma 5.10] to show that $G \leq A \Gamma L(1, v)$. This proves assertion (2). Assume that $n=2 v$. Then $v=2^{d}$ and $d$ is odd by [33, Theorem 13.18], and since $n$ is a square. Thus $d^{*}$ is odd, since $d^{*} \mid d$. Therefore, by Theorem 2.1, either $G \leq A \Gamma L\left(1,2^{d}\right)$ or $S L\left(d^{*}, 2^{h}\right) \unlhd G_{O}, d^{*} \geq 3$. Assume that the latter occurs. Let $\gamma$ be any involution of $G_{O}$ inducing a transvection on $\mathscr{O}$. Then $\gamma$ fixes $2^{d-h}$ points on $\mathscr{O}$ and the point in $l-\left(\mathscr{O} \cup \mathscr{O}^{\prime}\right)$. So $2^{2(d-h)} \leq 2^{d+1}$ by [33, Theorem 3.7], since $\gamma$ is a Baer collineation of $\Pi$ and $n=2^{d+1}$. This yields $2(d-h) \leq d+1$. Thus $d^{*}=3$ and $h=1$, since $d=d^{*} h$ and $d^{*} \geq 3$. Hence $S L(3,2) \leq G_{o}$ and $n=16$. This gives a contradiction by [15], since $|\mathscr{O}|=8$ and $G$ is of affine type. Thus $G \leq A \Gamma L\left(1,2^{d}\right)$ and we obtain assertion (3).

Assume that each involution in $G$ is a perspectivity of $\Pi$. If $n=2 v-1$, then each involution in $G$ must fix exactly 1 point on $\mathscr{O}$ and 1 point on $\mathscr{O}^{\prime}$, as $n$ is odd. Thus $v$ must be odd. Then either $G \leq A \Gamma L(1, v)$ or $S L\left(2, p^{h}\right) \unlhd G_{O}$ or $p^{d} \in\left\{3^{4}, 3^{6}, 19^{2}, 23^{2}\right\}$ 
by [28, Theorem 6.7], since $p^{d} \notin\left\{5^{2}, 7^{2}, 11^{2}, 29^{2}, 59^{2}\right\}$ by our assumption. Actually, the cases $p^{d} \in\left\{3^{4}, 3^{6}, 19^{2}, 23^{2}\right\}$ cannot occur by [33, Theorem 3.6]. Assume that $S L\left(2, p^{h}\right) \unlhd G_{O}$. Then there exists an element $\phi$ of order $p$ inducing a transvection on $\mathscr{O}$ and on $\mathscr{O}^{\prime}$. Then $\phi$ fixes $2 p^{h}$ points on $l$. Clearly $(n, p)=1$, since $n=2 v-1$. Furthermore $(n-1, p)=1$, since $p$ is odd. Therefore $\phi$ fixes a subplane of $\Pi$ of order $2 p^{h}-1$. Then $\left(2 p^{h}-1\right)^{2} \leq 2 p^{2 h}$ by [33, Theorem 3.7], which gives a contradiction. Thus $G \leq A \Gamma L(1, v)$ and this proves assertion (2). If $n=2 v$ then $v=2^{d}$ by [33, Theorem 13.18]. Then $G$ is solvable by [2, Satz 1]. In particular $G \leq A \Gamma L(1, v)$ by [35, Theorem XII.7.3]. That is the assertion (2).

Assume that $T \leq K \leq G$. Assume also that $|l-\mathscr{O}| \leq 2$. Then $|l-\mathscr{O}|=2$ and hence $n=p^{d}+1$, since $n>v$ and $v=p^{d}$. If 4||$G_{O} \mid$, then $G_{O}$ contains a Baer involution of $\Pi$, since $|l-\mathscr{O}|=2$. Thus $n$ must be a square. Clearly $d \geq 2$. Then $n=9$ and $v=8$ by [54, Result A5.1]. This gives a contradiction by [30, Theorem A]. Hence 2||$G_{o} \mid$ and $2^{2} \nmid\left|G_{0}\right|$. Then $G \leq A \Gamma L(1, v)$ by [28, Theorem 5.15]. Furthermore, either $p=2$ or $p^{d} \equiv 3 \bmod 4$, as $p^{d}-1|| G_{O} \mid$. This proves assertion (1).

Assume that $|l-\mathscr{O}|>2$ and that $v$ is even. Then each nontrivial element in $T$ is a Baer collineation of $\Pi$, since $|l-\mathscr{O}|>2$ and $T \leq K$. Thus $n+1-v=\sqrt{n}+1$, since $T$ fixes $l-\mathscr{O}$ pointwise and $T$ is regular on $\mathscr{O}$. Hence $v=\sqrt{n}(\sqrt{n}-1)$. This gives a contradiction, since $v=p^{d}$ and $v>4$. Hence $v$ is odd.

Assume that $G_{O}$ contains the involutory $O$-dilatation $\alpha$. Suppose that $T$ does not contain planar elements. If there exists $X \in \Pi-l$ such that $T_{X} \neq\langle 1\rangle$, then $T_{X}=T$, since $T$ is abelian and $T$ fixes $l-\mathscr{O}$ pointwise and $T$ does not contain any planar element. Thus $T$ is semiregular on $X Y-\{X, Y\}$ for any $Y \in l-\mathscr{O}$ again by the facts that $T$ fixes $l-\mathscr{O}$ pointwise and $T$ does not contain any planar element. Then $v \mid n-1$ and hence $n=v+1$, since $v<n \leq 2 v$. This gives a contradiction, since $|l-\mathscr{O}|>2$. Hence $T$ is semiregular on $\Pi-l$. In particular, $p \mid n$. Assume that $\alpha$ is a $\left(C_{\alpha}, a_{\alpha}\right)$-perspectivity of $\Pi$. Let $\gamma \in T, \gamma \neq 1$. Then $\alpha^{\gamma}$ is the $O \gamma$-involutory dilatation of $G$. Furthermore, $\alpha^{\gamma}$ is a $\left(C_{\alpha} \gamma, a_{\alpha} \gamma\right)$-perspectivity, where $C_{\alpha} \gamma \in l$ and $a_{\alpha} \gamma \neq l$. Clearly $\alpha^{\gamma} \neq \alpha$, as $O \gamma \neq O$. Then $\left\langle\alpha, \alpha^{\gamma}\right\rangle$ fixes $a_{\alpha} \cap a_{\alpha^{\gamma}}$ pointwise. This gives a contradiction, since $\left\langle\alpha, \alpha^{\gamma}\right\rangle \cap T \neq\langle 1\rangle$ and $\left(a_{\alpha} \cap a_{\alpha \gamma}\right) \cap(\Pi-l) \neq \emptyset$. Hence $\alpha$ is a Baer collineation of $\Pi$. Then $p \mid \sqrt{n}$, since $p \mid n$ and $n$ is a square. Assume that there exists a point $P \in l-\mathscr{O}$ such that $T$ is semiregular on $[P]-\{l\}$. Then $v \mid n$ and hence $n=2 v$, since $v<n \leq 2 v$. This gives a contradiction by [33, Theorem 13.18], since $v$ is odd. Hence, for each point $B \in l-\mathscr{O}$ there exists a line $r_{B} \in[B]-\{l\}$ such that $T_{r_{B}} \neq\langle 1\rangle$. Assume that there exists a point $D \in l-\mathscr{O}$ such that $D \alpha \neq D$. Then $T_{r_{D}}$ also fixes $r_{D \alpha}$, since $\alpha$ acts as the inversion on $T$. Thus $T_{r_{D}}$ fixes the point $r_{D} \cap r_{D \alpha}$ lying on $\Pi-l$. This gives a contradiction, since $T$ is semiregular on $\Pi-l$. As a consequence, $l \cap \operatorname{Fix}(\alpha)=(l-\mathscr{O}) \cup\{O\}$. Then $n+1-v=\sqrt{n}$, since $|l-\mathscr{O}|=n+1-v$. That is, $n-\sqrt{n}+1=v$. This gives 
a contradiction, since $p \mid \sqrt{n}$ and $v=p^{d}$. Hence $T$ contains a nontrivial planar element $\tau$.

Assume that $\alpha$ is a $\left(C_{\alpha}, a_{\alpha}\right)$-perspectivity. Then $C_{\alpha} \in l$ and $a_{\alpha} \neq l$, since $\alpha$ is the involutory $O$-dilatation in $G_{O}$ and $\mathscr{O} \subset l$. Note that $\left(C_{\alpha}, a_{\alpha}\right) \in \operatorname{Fix}(\tau)$, since $\alpha$ inverts $\tau$. So $\tau$ fixes $O$, since either $C_{\alpha}=O$ or $\{O\}=a_{\alpha} \cap l$. This gives a contradiction, since $O \in \mathscr{O}$, while $\tau$ is semiregular on $\mathscr{O}$.

Assume that $\alpha$ is a Baer collineation of II. Then $\alpha$ fixes $l-\mathscr{O}$ pointwise, since $\tau$ is planar and $\tau$ fixes $l-\mathscr{O}$ pointwise and $\alpha$ fixes only the point $O$ on $\mathscr{O}$. Thus $|l-\mathscr{O}|=\sqrt{n}$. That is $n+1-v=\sqrt{n}$ and hence $(\sqrt{n}-1)^{2}+(\sqrt{n}-1)+1=v$. Then either $v=p$ or $(\sqrt{n}-1, v)=\left(18,7^{3}\right)$ by [54, Result A7.1]. Assume that $(\sqrt{n}-1, v)=\left(18,7^{3}\right)$. Clearly $\alpha$ acts trivially on Fix $(\tau)$ by [33, Theorem 13.18], as $o(\operatorname{Fix}(\tau))=18$. Hence $\operatorname{Fix}(\tau) \subset \operatorname{Fix}(\alpha)$. This gives a contradiction by [33, Theorem 3.7], since $o(\operatorname{Fix}(\alpha))=19$. Therefore $v=p$ and we have assertion (4).

Assume that $G$ does not contain involutory dilatations. Then $S L\left(d^{*}, p^{h}\right) \unlhd G_{O}$ with $d^{*}$ odd by Theorem 2.1, since $v$ is odd. Let $\zeta$ be the involution in $G_{O}$ represented by the matrix $A=\operatorname{diag}\left(-I_{2}, I_{d^{*}-2}\right)$. Then $\zeta$ is a Baer collineation of $\Pi$ fixing exactly $p^{d-2 h}$ points on $\mathscr{O}$. Then $p^{2(d-2 h)} \leq n$, by [33, Theorem 3.7]. Thus $d^{*}=3$ as $d^{*}$ is odd and $d^{*}>1$. Let $L \leq C_{G_{o}}(\zeta)$, where $L=\langle\operatorname{diag}(C, 1): C \in S L(2, q)\rangle$. Let $L_{0}$ be the kernel of the action of $L$ on $\operatorname{Fix}(\zeta)$. Clearly $\langle\zeta\rangle \unlhd L_{0} \unlhd L$. Actually, $L_{0}<L$, otherwise $L$ would contain planar $p$-elements of $\Pi$ inducing transvections on $\mathscr{O}$ (for example pick $B=\operatorname{diag}\left(B_{0}, 1\right)$ where $B_{0}=\left(\begin{array}{ll}1 & 1 \\ 0 & 1\end{array}\right)$ ). Then $L_{0}=\langle\zeta\rangle$ and $L / L_{0} \cong P S L(2, q)$ acts on $\operatorname{Fix}(\zeta)$, fixing $q$ points on $\mathscr{O}$. It easily seen that $L / L_{0}$ contains a Baer collineation $\bar{\beta}$ of Fix $(\zeta)$ fixing a further point on Fix $(\zeta) \cap l-\mathscr{O}$, since $L / L_{0} \cong P S L(2, q)$ cannot be a group of perspectivities of Fix $(\zeta)$ with axis $\operatorname{Fix}(\zeta) \cap l$. Thus $o(\operatorname{Fix}(\zeta)) \geq q^{2}$ by [33, Theorem 3.7]. Then $n \geq q^{4}$, again by [33, Theorem 3.7]. This gives a contradiction, since $n \leq 2 q^{3}$ and $q$ is odd.

6.2. The unfaithful 2-transitive orbits. Throughout this subsection we assume that $N \neq\langle 1\rangle$.

The proof of Theorem 6.3, which is the main theorem in this subsection, is structured as follows. We firstly show that $G$ can be written in a 'nice' form (see the following lemma). Then we reduce to case $N \leq Z(G)$, otherwise Lemma 5.1 provides a lower bound for $|N|$ which is in contrast with the possible upper bounds given in Lemma 4.5. At this point we essentially use the Schur multipliers (see [41]) to obtain the assertion.

\section{LEMMA 6.2. The following hold:}

(1) $G=U G_{o}$, where $U$ is a normal p-subgroup $U$ of $G$ such that $U /(U \cap N) \cong \bar{T}$;

(2) if $G_{o}=H N$ with $H \cap N=\langle 1\rangle$ and $H$ quasisimple then $N \leq U$.

PROOF. Let $T$ be the full preimage in $G$ of $\bar{T}$. Then $T=U N$, where $U$ is a Sylow 
$p$-subgroup of $T$. Furthermore, $G=N_{G}(U) T$ by Frattini's argument. Actually $G=N_{G}(U)$, since $T=U N$ and $N=\Phi(G)$. Hence $U \triangleleft G$. Moreover, $U G_{O}$ induces $\bar{G}$ on $\mathscr{O}$, since $N<G_{O}$. Then $G=U G_{O}$ by the minimality of $G$. This proves assertion (1).

Assume that there exists $H \leq G_{O}$ such that $G_{O}=H N$ with $H \cap N=\langle 1\rangle$ and $H$ quasisimple. Then $G=U H$ by (1), since $N=\Phi(G)$. Furthermore, $U \cap H=\langle 1\rangle$, by [22, Theorem 3.1.3], since $H \cong \bar{G}_{O}$ and $U \cap H$ is a normal $p$-subgroup of $H$. Clearly $U N / U$ is isomorphic to a normal subgroup of $H$. Thus $U /(U \cap N)$ is isomorphic to a subgroup of $Z\left(\bar{G}_{O}\right)$, as $H \cong \bar{G}_{o}$ and the group $\bar{G}_{O}$ is quasisimple and $N$ is nilpotent. In particular $U /(U \cap N)$ has order coprime to $p$ by [22, Theorem 3.1.3]. Hence $N=P \times Z$, where $P=U \cap N$ and $Z$ is isomorphic to a subgroup of $Z\left(\bar{G}_{o}\right)$, as $N$ is nilpotent. In particular, $Z$ is cyclic because $Z\left(\bar{G}_{O}\right)$ is cyclic by [22, Theorem 3.2.1]. Let $W$ be the Sylow $t$-subgroup of $N$, with $t \neq p$. Then $W \leq Z$ as $N=P \times Z$. Thus $W$ is cyclic. Moreover, $W \triangleleft G$ as $N$ is nilpotent. Then $G$ acts on $W$ with kernel $Q$ and $G / Q \leq \operatorname{Aut}(W)$. Actually, $Q=G$ by [22, Theorem 1.3.10 and Lemma 5.4.1], since $W$ is cyclic and $N \leq Q$ and $G=U H$ with $H$ quasisimple. Thus $Z$ is central in $G$ and hence $U N=U \times Z$ as $(|U|,|Z|)=1$. This yields $U N \cap H=\langle 1\rangle$, since $U \cap H=\langle 1\rangle$ and $Z \cap H=\langle 1\rangle$. As a consequence, $Z=\langle 1\rangle$ and $N \leq U$. This proves assertion (2).

THEOREM 6.3. Let $\mathscr{J}=\left\{2^{4}, 2^{6}, 3^{2}, 3^{3}, 3^{4}, 3^{6}, 5^{2}, 7^{2}, 11^{2}, 19^{2}, 23^{2}, 29^{2}, 59^{2}\right\}$.

Then one of the following occurs:

(1) $\bar{G} \leq A \Gamma L(1, v)$, or

(2) $v \in \mathscr{J}$.

ProOF. Assume that the theorem does not hold. By Theorem 2.1, we have that if $\bar{G}=\bar{T} \bar{G}_{0}$ is 2-transitive on $\mathscr{O}$ then $\bar{G}=\bar{T} \operatorname{soc}\left(\bar{G}_{0}\right)$. Note that $\operatorname{soc}\left(\bar{G}_{0}\right)$ is still 2-transitive on $\mathscr{O}$ and $\operatorname{soc}\left(\bar{G}_{O}\right)$ is quasisimple. Thus, we may assume without loss of generality that $\bar{G}_{O}=\operatorname{soc}\left(\bar{G}_{o}\right)$. Hence $\bar{G}_{o}$ is quasisimple. In particular, we have the following possibilities for $\bar{G}_{o}$ :

(i) $\bar{G}_{O} \cong S L\left(d^{*}, p^{h}\right), d^{*} \geq 2$;

(ii) $\bar{G}_{O} \cong S p\left(d^{*}, p^{h}\right), d^{*}$ even and $d^{*} \geq 4$;

(iii) $\bar{G}_{o} \cong G_{2}\left(p^{h}\right), d^{*}=6$ and $p=2$.

We treat the cases $N \nsucceq Z(G)$ and $N \leq Z(G)$ separately.

(I) The case $N \npreceq Z(G)$.

Assume that $N \notin Z(G)$. Then, by the same argument as in Lemma 5.1, we deduce that $G$ acts on $V$ with kernel $R$, where $V=S / \Phi(S), S$ a Sylow $t$-subgroup of $N$ such that $S \succeq Z(G)$, and $N \unlhd R \unlhd G$. Assume that $R=G$. Then each Sylow $r$-subgroup $T$ of $G$, with $r \neq t$, centralizes $S$ by [22, Theorem 5.1.4]. 
Thus $C_{G}(S) \not \leq N$ and $\left[G: C_{G}(S)\right]=t^{h}, h \geq 0$. Furthermore, $C_{G}(S) \triangleleft G$ as $S \triangleleft G$. Hence $N \triangleleft C_{G}(S) N \unlhd G$. Set $L=C_{G}(S) N$. Clearly, either $L=G$ or $[G: L]=t^{i}$ for some $1 \leq i \leq h$. Actually, the former is ruled out by the same argument as in Lemma 5.1. Thus $[G: L]=t^{i}$ and hence $[\bar{G}: \bar{L}]=t^{i}$ as $N \triangleleft L$. Then $\bar{L} / \bar{T} \triangleleft \bar{G} / \bar{T}$ and $[\bar{G} / \bar{T}: \bar{L} / \bar{T}]=t^{i}$ as $\bar{T} \leq \bar{L}$ by [17, Theorem 4.3.B]. This implies that $\bar{G}_{o}$ must contain a normal subgroup of index $t^{i}$. This gives a contradiction, since $\bar{G}_{O}$ is quasisimple. Then $R<G$. Hence, either $R=N$ or $\bar{T} \leq \bar{R}<\bar{T} . Z\left(\bar{G}_{o}\right)$, since $\bar{G}=\bar{T} \bar{G}_{o}$.

Assume that $\bar{T} \leq \bar{R} \leq \bar{T} \cdot Z\left(\bar{G}_{o}\right)$. Set $H=G / R$. Clearly $H \leq P \Gamma L(V)$, since $V$ is a vector space over $G \bar{F}(t)$. Note that $H \cong \bar{G} / \bar{R}$, since $\bar{G} / \bar{R} \cong(\bar{G} / \bar{T}) /(\bar{R} / \bar{T})$. This implies that $H$ is isomorphic to a central extension of $\bar{G}_{o} / Z\left(\bar{G}_{o}\right)$, since $\bar{G} / \bar{T} \cong \bar{G}_{o}$ and since $\bar{R} / \bar{T}$ is isomorphic to a subgroup of $Z\left(\bar{G}_{o}\right)$. Thus $H \leq P S L(V)$, since $H \leq P \Gamma L(V)$ and $H$ is quasisimple. Recall that $\bar{G}_{o}$ is one of the groups listed above. So, if the representation is in coprime characteristic then $|V| \geq 2^{R_{p^{\prime}}(H)}$ by [43, Corollary 5.3.3, Theorem 5.3.9 and Corollary 5.4.14.(i)], since $v \notin \mathscr{J}$. This implies $|V|>4 v^{2}$ for $v>8$. Hence $|N|>4 v^{2}$ for $v>8$ as $V=S / \Phi(S)$ and $S$ is a Sylow $t$-subgroup of $N$. This gives a contradiction, since $|N| \leq 4 v^{2}$ as $|N| \leq n^{2}$ by Lemma 4.5 and $n \leq 2 v$. Hence $v \leq 8$. Actually $v=8$, since $v=p^{d}, d \geq 2$ and $v \geq 5$. In particular, $N$ is regular on $\Pi-l$ as $|N|=2^{6}$. Then $G=G_{C} N$ for some $C \in \Pi-l$. Actually $G=G_{C}$, since $N=\Phi(G)$ by Lemma 5.10. This gives a contradiction, since $N$ is semiregular on $\Pi-l$. Thus the representation of $H$ as a subgroup of $P S L(V)$ is in the natural characteristic. Therefore $v|| V \mid$ by [43, Corollary 5.3.3 and Proposition 5.4.13], since $\bar{G} \not \leq A \Gamma L(1, v)$ and $v \notin \mathscr{J}$. As a consequence, $v|| N \mid$ for $N<R<G$.

Finally, assume that $R=N$. Then $\bar{G} \leq P S L(V)$. Then, by the above argument with $\bar{G}_{O}, O \in \mathscr{O}$, in the role of $H$, we still obtain $v|| N \mid$. Hence $v|| N \mid$ in every admissible case.

Assume that $|N| \mid n-1$. Then $G$ fixes a unique point $Q$ on $\Pi-l$ and $N$ is semiregular on $\Pi-(l \cup\{Q\})$ by Lemma 4.5. Then $|N|=n-1$, since $v|| N \mid$ and $v<n \leq 2 v$. Thus $G_{O}=G_{O, A} N$ with $G_{O, A} \cap N=1$, for some point $A \in O Q-\{O, Q\}$. Then $G=U G_{O, A}$ by Lemma 6.2, as $G=U G_{O}, G_{O}=G_{O, A} N$ and $N=\Phi(G)$. Then the argument of Theorem 6.1 , with $\bar{G}$ in the role of $G$, rules out this case, since $\bar{G} \not \subset A \Gamma L(1, v), v \notin \mathscr{J}$ and $v=p^{d}$.

Assume that $|N| \mid n$. Then $|N|=n$ and $n=2 v$ since $v|| N \mid$ and $v<n \leq$ $2 v$. Therefore $v=2^{d}$ and $n=2^{d+1}$ by [33, Theorem 13.18]. Furthermore, $N$ is semiregular on $[O]-\{l\}$ where $O \in \mathscr{O}$ by Lemma 4.5. Let $\Omega$ be the set of $N$ orbits on $[O]-\{l\}$. Clearly $|\Omega| \leq 2$, since $v|| N \mid$ and $n=2 v$. Thus $G_{O}$ fixes $\Omega$ elementwise. Then $G_{O}=G_{o, a} N$ for some line $a \in[O]-\{l\}$ and the argument of Theorem 6.1, with $G_{O, a}$ in the role of $G_{O}$, rules out the case $d$ odd. Hence $d$ is even and $n$ is a non square as $n=2^{d+1}$. Thus $G_{o, a}$ must have odd order, since $v$ and $n$ 
are even and $G_{o, a} \cong \bar{G}_{o}$. Therefore $\bar{G}_{o}$ must have odd order. Hence $G$ is solvable by [2, Satz 1]. In particular, $G \leq A \Gamma L(1, v)$ by [35, Theorem XII.7.3], which is a contradiction.

Assume that $|N|>n$. Then either $n|| N \mid$ and $n$ is a prime power, or $n=3|J| / 2$ where $J$ is the Sylow 2 -subgroup of $N$ by Lemma 4.5. Then either $n=2 v$, or $n=3 v / 2$ and $v$ even, since $v \mid n^{2}$ as $|N| \mid n^{2}$, and since $v<n \leq 2 v$, respectively. Note that $v$ is also even in the first case by [33, Theorem 13.18]. Let $\Omega$ be defined as above. Then $|\Omega| \leq 3$, since each $N$-orbit on $[O]-\{l\}$ has length at least $n / 3$ by Lemma 4.5 . Thus $G_{O}$ fixes $\Omega$ elementwise, since $G_{O}$ is quasisimple. Hence $G_{o}=G_{o, b} N$ for some line $b \in[O]-\{l\}$. We stress that $G_{o, b} \cap N \neq\langle 1\rangle$, since $N_{b} \neq\langle 1\rangle$ as $|N|>n$. Now, we may repeat the above argument, with $G_{O, b}$ in the role of $H$ and $N_{b}$ in the role of $N$, to assert that either $v|| N_{b} \mid$ or $N_{b} \leq Z\left(G_{O . b}\right)$. Assume that $v|| N_{b} \mid$. Then $|N| \geq v n / 3$, since $\left[N: N_{b}\right] \geq n / 3$ as $|\Omega| \leq 3$. Let $\Psi$ be the set of $N$-orbits of points on $\Pi-l$. Then $|\Psi| \leq 6$, since $N$ is semiregular on $\Pi-l$ and $|N| \geq v n / 3$. It is a plain to see that $G$ fixes $\Psi$ elementwise, since $v \geq 8$. So $G=G_{A} N$ for some $A \in \Pi-l$. Actually $G=G_{A}$, since $N=\Phi(G)$ by Lemma 5.10. This gives a contradiction, since $N$ is semiregular on $\Pi-l$. Hence $N_{b} \leq Z\left(G_{O, b}\right)$. Then $G_{O, b}=G_{O, b}^{\prime} N$ where $G_{o, b}^{\prime}$ is a covering group for $\bar{G}_{O}$ by [1, Theorem 11.3.33]. Hence, $G_{O, b}^{\prime} \cap N$ is isomorphic to a subgroup of the Schur multiplier of $\bar{G}_{o}$. If $\bar{G}_{O} \cong S L\left(d^{*}, p^{h}\right), d^{*} \geq 2$, then $G_{O, b}^{\prime} \cap N=\langle 1\rangle$ by [41, Theorem 7.1.1 (i)], since $v \notin \mathscr{J}$. If $\bar{G}_{o} \cong S p\left(d^{*}, p^{h}\right), d^{*}$ even, $d^{*} \geq 4$, then $G_{O, b}^{\prime} \cap N=\langle 1\rangle$ by [ 41 , Theorem 2.5.12] when $p$ is odd, and by [43, Theorem 5.1.4] when $p=2$, since $\bar{G}_{O}$ is perfect, $d^{*} \geq 4$ and $v \notin \mathscr{J}$. Finally, if $\bar{G}_{O} \cong G_{2}\left(p^{h}\right)$ and $p=2$, then $G_{O, b}^{\prime} \cap N=\langle 1\rangle$ by [43], Theorem 5.1.4, since $v \notin \mathscr{J}$. Thus $G_{O, b}^{\prime} \cap N=\langle 1\rangle$ in every admissible case. Then the above argument, with $G_{O, b}^{\prime}$ in the role of $G_{o, a}$, rules out this case. Actually, such an argument works when we replace $n=2 v$ with $n=3 v / 2$. So, also the case $n=3 v / 2$ cannot occur.

(II) The case $N \leq Z(G)$.

Assume that $N \leq Z(G)$. Then $G_{o}=G_{o}^{\prime} N$ with $G_{o}^{\prime}$ a covering group for $\bar{G}_{o}$ by [1, Theorem 11.3.33]. Hence $G_{O}^{\prime} \cap N$ is isomorphic to a subgroup of the Schur multiplier of $\bar{G}_{o}$. Actually, $G_{o}^{\prime} \cap N=\langle 1\rangle$ in every admissible case by the above argument, with $G_{o}^{\prime}$ in the role of $G_{o, b}^{\prime}$. Then $N \leq \dot{U}$ by Lemma 6.2(2). Furthermore, there exists $\zeta \in G_{O}^{\prime}$ such that $o(\zeta)$ is a primitive prime divisor of $v-1$ by [43, Theorem 5.2.14], since $\bar{G} \not \leq A \Gamma L(1, v)$ and $v \notin \mathscr{J}$. Then $\zeta$ acts irreducibly on $U /(U \cap N) \cong \bar{T}$ by [28, Section 5 and Theorem 3.5] and each proper $\zeta$-invariant normal subgroup of $U$ lies in $N$. Therefore $\zeta$ acts trivially on each proper $\zeta$-invariant normal subgroup of $U$, since $N=Z\left(G_{o}\right)$. Then $U$ is special and $N=\Phi(U)$ by [22, Theorem 5.3.7], since $U / N \cong \bar{T}$ and $N \neq\langle 1\rangle$. Recall that $K$ is the kernel of the representation of $G$ on $l-\mathscr{O}$ and $N=\Phi(G)$. If $K<G$ then there exists a nontrivial 
$G$-orbit $\mathscr{O}^{*}$ on $l-\mathscr{O}$. Let $\Lambda$ be the set of $N$-orbits on $\mathscr{O}^{*}$ and let $F$ be the kernel of the representation of $G$ on $\Lambda$. Clearly $N \unlhd F \unlhd G$. If $F=G$ then $G=G_{X} N$ for some $X \in \mathscr{O}^{*}$. That is $G=G_{X}$, since $N=\Phi(G)$. This gives a contradiction, since $X \in \mathscr{O}^{*}$ and $\mathscr{O}^{*}$ is a nontrivial $G$-orbit. Therefore $R<G$.

If $R=N$ then $G$ induces $\bar{G}$ on $\mathscr{O}^{*}$. Then $v|| \mathscr{O}^{*} \mid$ by the $\mathrm{O}^{\prime}$ Nan-Scott Theorem, since $v=|\bar{T}|$. This forces $N$ to fix $l-\mathscr{O}$ pointwise. Then either $n=2 v-1$ and $N=N(Q, l)$ with $Q \in \Pi-l$, or $n=2 v$ and $N=N(l, l)$ by Lemma 4.5, since $n \leq 2 v$. Since $\bar{G} \not \subset A \Gamma L(1, v)$ and $v \notin \mathscr{J}$, and since $G_{o}^{\prime} \cap N=\langle 1\rangle$ and $G_{O}^{\prime} \cong \bar{G}_{O}$, the arguments of Theorem 6.1 still work and hence we may rule out the cases $n=2 v-1$ and $n=2 v$.

Now, assume that $N<R<G$. That is $\langle 1\rangle<\bar{R} \triangleleft \bar{G}$. Hence $U \leq R$, by [17, Theorem 43.B], since $N \leq U, \bar{U}=\bar{T}$ and $\bar{T}=\operatorname{soc}(\bar{G})$. Thus $U$ fixes $\Lambda$ elementwise. Then $U=U_{Y} N$ for each $Y \in \mathscr{O}^{*}$, since $N \leq U$. Furthermore, $U$ fixes $\mathscr{O}^{*}$ pointwise since $N=\Phi(U)$. Hence $N<U \leq K \leq G$ and $N=N(l, l)$ or $N(Q, l)$ with $Q \in \Pi-l$ by Lemma 4.5. Assume that $N=N(l, l)$. Clearly $N(X, l)=\langle 1\rangle$ for any $X \in \mathscr{O}$, since $N \leq Z(G)$. Hence there exists a point $E \in l-\mathscr{O}$ such that $N(E, l) \neq\langle 1\rangle$, since $N \neq\langle 1\rangle$.

Assume that $N(E, l)<N$. Then $G$ acts on the set $\Sigma$ of $N$-orbits on $[E]-\{l\}$, since $N \leq Z(G)$. If $U$ fixes some element in $\Sigma$ then $U=U_{r} N$ for some $r \in[E]-\{l\}$, since $N \leq U$. As a consequence, $U=U_{r}$ since $N=\Phi(U)$. This gives a contradiction, since $N_{r}=N(E, l)$ and $N(E, l)<N$. Thus $U$ moves each element in $\Sigma$. In particular, $G$ induces $\bar{G}$ on $\Sigma$, and $\bar{G}$ does not fix any element in $\Sigma$. Then $v|| \Sigma \mid$ by the O'Nan-Scott Theorem, since $v=|\bar{T}|$. Then $n=v[N: N(E, l)]$, since $|\Sigma|=n /[N: N(E, l)]$. Thus $[N: N(E, l)]=2$ and $n=2 v$, since $v<n \leq 2 v$. Since $\bar{G} \not \leq A \Gamma L(1, v), v \notin \mathscr{J}$ and since $G_{o}^{\prime} \cap N=\langle 1\rangle$ and $G_{O}^{\prime} \cong \bar{G}_{O}$, it is easily seen that the argument of Theorem 6.1 still works and we may again rule out the case $n=2 v$. Therefore $N=N(E, l)$.

Assume that $v$ is even. If there exists an involution $\sigma \in U-N$ then $\sigma$ is a Baer collineation of $\Pi$ fixing the $v+1$ points of $l-\mathscr{O}$, since $n=2 v$ and since $U$ fixes $l-\mathscr{O}$ pointwise. Then $v^{2} \leq n$ by [33, Theorem 3.7]. This gives a contradiction, since $n=2 v$ and $v>2$. As a consequence $U-N$ does not contain involutions. Thus $U$ must be semiregular on $[Y]-\{l\}$ for any point $Y$ on $l-(\mathscr{O} \cup\{E\})$, since $U$ fixes $l-\mathscr{O}$ pointwise, $N \triangleleft U$ and $N=N(E, l)$. Thus $|U| \mid n$. Then $n=2 v$ and $N \cong Z_{2}$, since $|U|=v|N|$ and $N \neq\langle 1\rangle$. Hence $v$ is even as $N \leq U$ and $U$ is a $p$-group. This gives a contradiction by the same argument as above. Assume that $v$ is odd. Assume also that $U$ fixes a line $f$ of $[E]-\{l\}$. If $U$ is semiregular on $f-\{E\}$, then $n=2 v$, since $|U|=v|N|$. This gives a contradiction by [33, Theorem 13.18], since $v$ is odd. Thus $U_{D} \neq\langle 1\rangle$ for some $D \in f-\{E\}$. Then $U_{D}$ fixes $D^{N}$ pointwise, since $N \leq Z(G)$. Then there exists a non trivial element $\tau$ in $U-N$ fixing $D^{N} \cup(l-\mathscr{O})$ pointwise. Thus $\tau$ is planar on $\Pi$, since $|l-\mathscr{O}| \geq 2,\left|D^{N}\right| \geq 2$ and $D^{N} \subset \Pi-l$. 
So $o(\operatorname{Fix}(\tau))=n-v$. If $n+1-v=\sqrt{n}$ then, by arguing as in Theorem 6.1, we deduce that $v$ is a prime. This gives a contradiction, since $\bar{G} \not \leq A \Gamma L(1, v)$.

Assume that $v$ is odd. So, $n+1-v<\sqrt{n}$. In particular, $n \leq v+\sqrt{2 v}$ by [33, Theorem 3.7], since $n \leq 2 v$. Recall that $G_{O}^{\prime} \cap N=\langle 1\rangle$. The argument of Theorem 6.1 implies that $G_{O}^{\prime}$ contains an involution $\alpha$ inducing an involutory $O$-dilatation on $\mathscr{O}$. So $v$ is a square and hence $n$ cannot be a square, since $v<n \leq v+\sqrt{2 v}$. Clearly $\alpha$ cannot be a Baer collineation of $\Pi$. Thus $\alpha$ is an involutory $\left(C_{\alpha}, a_{\alpha}\right)$ perspectivity. Clearly $C_{\alpha} \in l$ and $a_{\alpha} \neq l$ since $\alpha \notin N$ and $\alpha$ fixes $l$. Thus there exists a point $W$ on $l-\mathscr{O}$ such that $\left|W^{H}\right|>1$. Let $\Gamma$ be the set of $\alpha$-orbits on $W^{H}$. Then $|\Gamma|=\left|W^{H}\right| / 2$, since $\alpha$ is central in $H, a_{\alpha} \neq l$ and $H$ is transitive on $W^{H}$. Then $|\Gamma| \geq d_{0}(H / Z(H))$ by [43, Proposition 5.2.1]. Hence $2 d_{0}(H / Z(H)) \leq n-v+1$, since $|\Gamma|=\left|W^{H}\right| / 2$ and $\left|W^{H}\right| \leq n-v+1$. Actually, $\left(2 d_{0}(H / Z(H))-1\right)^{2} \leq 2 v$, since $v<n \leq v+\sqrt{2 v}$. At this point, bearing in mind that $d_{0}(H / Z(H))$ is given in [12], it is a straightforward calculation to show that no case satisfies the previous inequality. Hence $U$ does not fix lines of $[E]-\{l\}$. Therefore $[E]-\{l\}$ is a union of nontrivial $\bar{G}$-orbits. Thus $v \mid n$, since the length of each these orbits is a multiple of $v$ by the O'Nan-Scott Theorem, as $v=|\bar{T}|$. Hence $n=2 v$. Again this gives a contradiction.

Assume $N=N(Q, l)$ for some $Q \in \Pi-l$. Then $N$ is semiregular on $Q B-\{Q, B\}$, where $B$ is any point of $l-\mathscr{O}$. If $U$ is semiregular on $Q B-\{Q, B\}$, then $|U| \mid n-1$. Then $2 v \leq n-1$, since $|U|=v|N|$ and $N \neq\langle 1\rangle$. This gives a contradiction, since $n \leq 2 v$. In particular there exists $\tau_{1} \in U-N$ fixing a point $C$ of $Q B-\{Q, B\}$. Then $\tau_{1}$ is planar on $\Pi$, since $\tau_{1}$ fixes the points $C$ and $Q$ on $\Pi-l$ and since $U$ fixes $l-\mathscr{O}$ pointwise. At this point the same argument used for the case $N=N(E, l)$, with $\tau_{1}$ in the role of $\tau$, still works and we may rule out this case. This completes the proof.

\section{Translation Planes}

In this section we investigate what Theorems 1.1 and 1.2 say when $\Pi$ is the projective extension of a translation plane of order $n$ and $\mathscr{O}$ is a 2-transitive $G$-orbit of length $v$ on a line $l$, with $n>v \geq n / 2$. The case when $\mathscr{O}$ has length $v$ with $v \geq n$ is already contained in Section 2.

THEOREM 7.1. Let $\Pi$ be the projective extension of a translation plane of order $n$ and let $O$ be a 2-transitive $G$-orbit of length $v$ on a line l. If $n>v \geq n / 2$ and $G$ is almost simple, then one of the following occurs:

(1) $\Pi$ is the Hall plane of order 9 or its dual, $|\mathscr{O}|=5$ and $S L(2,5) \unlhd G$, in particular $l$ is the line at infinity;

(2) $\Pi$ is the Johnson-Walker translation plane of order 16 or its dual, and $P S L(2,7) \unlhd G$. In particularl is an affine line. 
PROOF. Suppose that $\Pi$ is the projective extension of a translation plane of order $n$. Then $n=t^{j}$ where $t$ is a prime and $j \geq 1$. Assume that $n=2 q+1, q \equiv 3 \bmod 4$, $q \neq 7,|\mathscr{O}|=q+1$ and $S L(2, q) \unlhd G$. Then $j=1$ by Lemma $4.1(1)$, so $\Pi$ is Desarguesian. Then $P S L(2, q) \leq P G L(2, n)$, since $G$ induces the group $P S L(2, q)$ on $l$. Since $P S L(2, q)$ contains nontrivial elements fixing 4 points on $l$, namely two points on $\mathscr{O}$ and two points on $\mathscr{O}^{\prime}$, we have a contradiction. Now, assume that $n=2(q+1), q \equiv 3 \bmod 4,|\mathscr{O}|=q+1$ and $S L(2, q) \unlhd G$. Then $n=2^{j}$ and $q$ is a Mersenne prime by Lemma 4.1(1). Clearly $G \leq P \Gamma L\left(2 h, 2^{j_{1}}\right)$, with $j=j_{1} h$, since $G$ leaves invariant the line at infinity. Thus $S L(2, q) \leq P S L\left(2 h, 2^{j_{1}}\right)$, since $S L(2, q) \unlhd G$. Then $2 h>(q-1) / 2$ by [43, Proposition 5.3.2 and Theorem 5.3.9], since $q \neq 5,9$ as $q$ is a Mersenne prime. Hence $2(q+1) \geq 2^{(q-1) / 4}$, since $n=2^{j}$ and $n=2(q+1)$. An easy computation shows that the previous inequality is impossible for $q>19$, since $q$ is odd. Hence $q \leq 19$. Actually $q=7$, since $q$ must be a Mersenne prime. Thus $S L(2,7) \unlhd G$ and $n=16$. A direct inspection of the list of the full collineation groups of all translation planes of order 16 given in [16] (see also [53]) rules out this case. So, the cases (2b) and (3b) of Theorem 1.1 cannot occur when $\Pi$ is the projective extension of a translation plane of order $n$. Thus the assertion follows by Theorem 1.1 , since $n>v \geq n / 2$.

THEOREM 7.2. Let $\Pi$ be the projective extension of a translation plane of order $n$ and let $\mathscr{O}$ be a 2-transitive $G$-orbit of length $v$ on a line $l$, with $n>v \geq n / 2$. If $G$ is of affine type and $G$ acts faithfully on $\mathscr{O}$ and $v \notin\left\{5^{2}, 7^{2}, 11^{2}, 29^{2}, 59^{2}\right\}$, then $G \leq A \Gamma L(1, v)$. Furthermore one of the following occurs:

(1) $n=2 v-1, v=p^{d}$, in particular, either $\left(p^{d}, n\right)=\left(13^{4}, 239^{2}\right)$ or $n=t^{2^{5}}$ and $d \leq 2$;

(2) $n=2 v, v=2^{d}$.

PROOF. Suppose that $\Pi$ is the projective extension of a translation plane. Then $n=t^{j}, j \geq 1$, for some prime $t$. Assume that $\mathscr{O}$ is a 2-transitive $G$-orbit of length $v$ on a line, with $n>v \geq n / 2$ and $v \notin\left\{5^{2}, 7^{2}, 11^{2}, 29^{2}, 59^{2}\right\}$. Assume also that $G$ is a collineation group of $\Pi$ of affine type acting faithfully on $\mathscr{O}$. Then $G \leq A \Gamma L(1, v)$ and $n$ satisfies one of the relations (1)-(4) given in Theorem 6.1. Assume that $n=v+1$ with $v=2^{d}$ or $v \equiv 3 \bmod 4$. If $v=2^{d}$ then either $d=3$ and $n=9$ or $n$ is a Fermat prime by [54, Result (B1.1)]. If $n=9$, then $\Pi$ is either Desarguesian or one of the Hall planes. Nevertheless these planes cannot occur since 7||$G \mid$, as $G$ is 2-transitive on $\mathscr{O}, \mathscr{O} \subset l$ and $v=8$. Thus $n$ is a Fermat prime and hence $\Pi$ is Desarguesian. Then $G \leq P G L(2, n)$. Thus $2^{d}\left(2^{d}-1\right) \mid 2^{d}\left(2^{d}+1\right)\left(2^{d}+2\right)$, since $G$ is 2 -transitive on $\mathscr{O}$ and $n=2^{d}+1$. This gives a contradiction, since $n>5$ by our assumptions.

Assume that $n-\sqrt{n}+1=v$ and $v=p$. Then $G=A G L(1, p)$. In this case the group $G$ fixes $l-\mathscr{O}$ pointwise and the element $\rho$ in $G$ of order $p$ is planar by 
Theorem 6.1 (see its proof). Hence $\rho$ fixes a subplane of $\Pi$ of order $t^{j}-p$ as $n=t^{j}$, $j \geq 1$. Then $t^{j}-p \mid t^{j}$ as $\Pi$ is a translation plane. So $t=p=2$ and $n=t^{j}=4$. This gives a contradiction, since $n-\sqrt{n}+1=p$. Thus either $n=2 v-1$ with $v=p^{d}$, or $n=2 v$ with $v=2^{d}$ by Theorem 6.1. The second case leads to assertion (2). Hence, assume that $n=2 v-1$ with $v=p^{d}$. Then $2 p^{d}=t^{j}+1$ as $n=t^{j}$. Assume that $j$ is not a power of 2 . Then $t^{h}+1 \mid t^{j}+1$ for some integer $1 \leq h<j$. Then $p \mid t^{h}+1$ and hence $t^{j}+1$ has no primitive prime divisors for $j>1$, as $2 p^{d}=t^{j}+1$. Then $t^{j}=8$ by [54, Result (P1.7)(ii)]. This gives a contradiction, since $t$ must be odd. Therefore $j=1$ and hence $t=2 p^{d}-1$, so $\Pi$ is Desarguesian. Then $G \leq P G L(2, t)$, since $G$ leaves invariant $l$. Since $p^{d}=(t+1) / 2$ and the Sylow $p$-subgroup of $G$ is normal in $G$, we have $G \leq N_{P G L(2, t)}\left(Z_{t+1}\right)$. So $p^{d}\left(p^{d}-1\right) \mid 2(t+1)$, since $G$ is 2-transitive on $\mathscr{O}$. Then $p^{d}-1 \mid 4$, since $t=2 p^{d}-1$. Hence $p^{d}=5$ and $n=9$, since $v \geq 5$. This gives a contradiction, since $n=t$ with $t$ prime. Hence $j=2^{h}, h \geq 0$. If $d>2$, then $\left(p^{d}, t^{j}\right)=\left(13^{4}, 239^{2}\right)$ by [54, Result (A11.1) and result of Page 141]. This proves assertion (1).

We remark that, while there are no known examples corresponding to case (1) of the previous theorem, Example 3 is an example for case (2). Indeed (2) and (6) in Example 1 do not correspond to case (1) of the previous theorem but correspond to particular cases of Theorem 6.3. Finally, we remark that there are no improvements to Theorem 6.3 when $\Pi$ is the projective extension of a translation plane.

\section{References}

[1] M. Aschbacher, Finite group theory (Cambridge University Press, 1996).

[2] H. Bender, 'Endliche zweifach transitive permutationsgruppen, deren involutionen keine fixpunkte haben', Math. Z. 104 (1968), 175-204.

[3] _ - 'Transitive gruppen gerader ordnung, in denen jede involution genau einen puntk festläß t', J. Algebra 17 (1971), 527-554.

[4] M. Biliotti and E. Francot, 'Two-transitive orbits in finite projective planes', J. Geom. 82 (2005), $1-24$.

[5] M. Biliotti, V. Jha and N. L. Johnson, 'The collineation group of generalized twisted fields planes', Geom. Dedicata 76 (1999), 97-126.

[6] M. Biliotti and N. L. Johnson, 'The non-solvable rank 3 affine planes', J. Combin. Theory Ser. A 93 (2000), 201-230.

[7] M. Biliotti and G. Korchmáros, 'Some new results on collineation groups preserving an oval of a finite projective plane', in: Combinatorics '88, Vol. 1 (Ravello, 1988), Res. Lecture Notes Math., Mediterranean, Rende (1991) pp. 159-170.

[8] M. Biliotti and A. Montinaro, 'Finite projective planes of order $n$ with a 2-transitive orbit of length $n-3$ ', Adv. Geom. 6 (2005), 15-37.

[9] J. Cofman, 'Double transitivity in finite affine and projective planes', Atti Accad. Naz. Lincei, Rend. Cl. Sci. Fis. Mat. Nat. 8 (1967), 317-320.

[10] — 'On a conjecture of Hughes', Proc. Camb. Phil. Soc. 63 (1967), 647-652. 
[11] J. H. Conway, R. T. Curtis, R. A. Parker and R. A. Wilson, Atlas of Finite Groups. Maximal subgroups and ordinary characters for simple groups (Oxford University Press, 1985).

[12] B.N. Cooperstein, 'Minimal degree for a permutation representation of a classical group', Israel J. Math. 30 (1978), 213-235.

[13] T. Czerwinski, 'Finite translation planes with a collineation groups doubly transitive on the points at infinity', J. Algebra 22 (1972), 428-441.

[14] — , 'On collineation groups that fix a line of a finite projective plane', Illinois J. Math. 16 (1977), 221-230.

[15] U. Dempwolff, 'The projective planes of order 16 admitting $S L(3,2)$ ', Rad. Mat. 7 (1991), 123134.

[16] U. Dempwolff and A. Reifart, 'The classification of the translation planes of order 16. I', Geom. Dedicata 15 (1984), 137-153.

[17] J. D. Dixon and B. Mortimer, Permutation groups (Springer Verlag, New York, 1966).

[18] D. A. Foulser, 'Solvable flag transitive affine groups', Math. Z. 86 (1964), 191-204.

[19] M. J. Ganley and V. Jha, 'On translation planes with a 2-transitive orbit on the line at infinity', Arch. Math. (Basel) 47 (1986), 379-384.

[20] M. J. Ganley, V. Jha and N. L. Johnson, 'The translation planes admitting a nonsolvable doubly transitive line-sized orbit', J. Geom. 69 (2000), 88-109.

[21] G. Glauberman, 'Central elements in core-free groups', J. Algebra 4 (1966), 403-420.

[22] D. Gorenstein, Finite groups (Chelsea Publishing Company, New York, 1980).

[23] D. Gorenstein and J. H. Walter, 'The characterization of finite groups with dihedral Sylow 2subgroups I', J. Algebra 2 (1965), 85-151.

[24] The GAP Group, 'Gap - groups, algorithms, and programming, version 4.3', http://www.gapsystem.org, 2002.

[25] R. W. Hartley, 'Determination of the ternary collineation groups whose coefficients lie in $G F\left(2^{n}\right)^{\text {', }}$ Ann. Math. 27 (1926), 140-158.

[26] C. Hering, 'Eine bemerkung über automorphismengruppen von endlichen projektiven ebenen und möbiusebenen', Arch. Math. (Basel) 18 (1967), 107-110.

[27] _ ' 'On involutorial elations of projective planes', Math. Z. 132 (1973), 91-97.

[28] - 'Transitive linear groups and linear groups which contain irreducible subgroups of prime order', Geom. Dedicata 2 (1974), 425-460.

[29] Y. Hiramine, 'On finite affine planes with a 2-transitive orbit on $l_{\infty}$ ', J. Algebra 162 (1993), $392-409$.

[30] C. Y. Ho, 'Involutory collineations of finite planes', Math. Z. 193 (1986), 235-240.

[31] - 'Projective planes of order 15 and other odd composite orders', Geom. Dedicata 27 (1988), $49-64$.

[32] C. Y. Ho and A. Gonçalves, 'On totally irregular simple collineation groups', in: Advances in finite geometries and designs (Chelwood Gate 1990) (eds. J. W. P. Hirschfeld, P. R. Hughes and J. A. Thas), Oxford Sci. Publ. (Oxford Univ. Press, New York, 1991) pp. 177-193.

[33] D. R. Hughes and F. C. Piper, Projective Planes (Springer Verlag, New York - Berlin, 1973).

[34] B. Huppert, Endliche Gruppen I (Springer Verlag, New York - Berlin, 1967).

[35] B. Huppert and N. Blackburn, Finite Groups III (Springer Verlag, Berlin - Heidelberg - New York, 1982).

[36] Z. Janko and T. Van Trung, 'The full collineation group of any projective plane of order 12 is a \{2, 3\}-group', Geom. Dedicata 12 (1982), 101-110.

[37] N. L. Johnson, 'A note on the derived semifield planes of order 16', Aequationes Math. 18 (1978), 103-111.

[38] M. Kallaher, 'Translation planes", in: Handbook Of Incidence Geometry (ed. F. Buekenhout) (Elsevier, 1995) pp. 137-192. 
[39] W. M. Kantor, 'On unitary polarities of finite projective planes', Canad. J. Math. 23 (1971), 1060-1077.

[40] —_, 'Homogeneous designs and geometric lattices', J. Combin. Theory Ser. A 38 (1985), 66-74.

[41] G. Karpilovsky, The Schur Multiplier (Clarendon Press, Oxford, 1987).

[42] P. B. Kleidman, 'The maximal subgroups of the chevalley groups $G_{2}(q)$ with $q$ odd, the Ree groups ${ }^{2} G_{2}(q)$, and their automorphism groups', J. Algebra 117 (1988), 30-71.

[43] P. B. Kleidman and M. Liebeck, The subgroup structure of the finite classical groups (Cambridge University Press, Cambridge, 1990).

[44] G. Korchmáros, 'Collineation groups doubly transitive on the points at infinity in an affine plane of order $2^{r}$ ', Arch. Math. (Basel) 37 (1981), 572-576.

[45] X. Li, 'A characterization of the finite simple groups', J. Algebra 245 (2001), 620-649.

[46] M. W. Liebeck, 'On the order of maximal subgroups of the finite classical groups', Proc. London Math. Soc. (3) 50 (1985), 426-446.

[47] I. Matulić-Bedenić, 'The classification of projective planes of order 11 which possess an involution', Rad. Mat. 1 (1985), 149-157.

[48] — - 'The classification of projective planes of order 13 which possess an involution', Rad Hrvatske Akad. Znam. Umjet. 456 (1991), 9-13.

[49] H. H. Mitchell, 'Determination of ordinary and modular ternary linear groups', Trans. Amer. Math. Soc. 12 (1911), 207-242.

[50] B. Mwene, 'On the subgroups of the group $P S L_{4}\left(2^{m}\right)$ ', J. Algebra 41 (1976), 79-107.

[51] D. S. Passman, Permutation Groups (W. A. Benjamin, Inc., New York -Amsterdam, 1968).

[52] T. Penttila, G. F. Royle and M. K. Simpson, 'Hyperovals in the known projective planes of order 16', J. Combin. Des. 4 (1996), 59-65.

[53] A. Reifart, 'The classification of the translation planes of order 16. II', Geom. Dedicata 17 (1984), 1-9.

[54] P. Ribenboim, Catalan's conjecture (Acad. Press, Boston, 1994).

[55] _ Fermat's last theorem for amateurs (Springer-Verlag, New York, 1999).

[56] R. H. Schulz, 'Über translationsebenen mit kollineationsgruppen, die die punkte der ausgezeichneten geraden zweinfach transitiv permutieren', Math. Z. 122 (1971), 246-266.

[57] R. Shull, 'Collineations of projective planes of order 9', J. Combin. Theory Ser. A 37 (1984), 99-120.

[58] — 'The classification of projective planes of order 9 possessing a collineation group of order 5', Algebras Groups Geom. 2 (1985), 365-379.

[59] M. Suzuki, 'On a class of doubly transitive groups', Ann. of Math. (2) 75 (1962), 105-145.

[60] L. Yu and M. Le, 'On the diophantine equation $\left(x^{n}-1\right) /(x-1)=y^{m}$ ', Acta Arith. 21 (1972), 299-301.

Dipartimento di Matematica, Università degli Studi di Lecce

Via per Arnesano

73100 Lecce

Italy

e-mail: alessandro.montinaro@unile.it 
